\title{
Digital appendix 5. Fiction syndicates in provincial newspapers, 1877 to 1899
}

$\mathrm{X}-$ Extended fiction discovered through paratextual method

$\mathrm{T}$ - Extended fiction in Trove but discovered manually (while exploring digitized archive for evidence of syndication)

\# Author probably Australian but not proven to be so

NB: Subtitles are omitted; refer to curated dataset for full publication details; years refer to when publication began; split years indicate publication began in both years, in different newspapers

\section{Syndicate 1}

\begin{tabular}{|c|c|c|c|c|c|c|c|c|c|c|c|c|c|c|c|c|c|c|c|c|c|c|c|c|c|c|c|c|c|c|c|c|c|c|c|c|c|}
\hline Title & Author & Nat. & 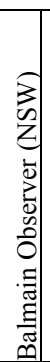 & 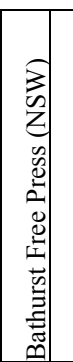 & 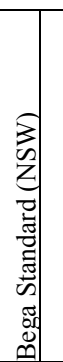 & 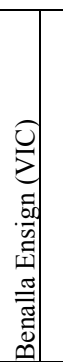 & 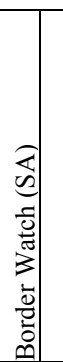 & 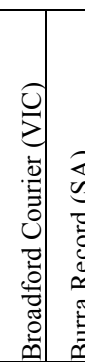 & 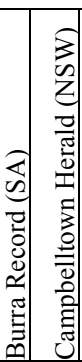 & 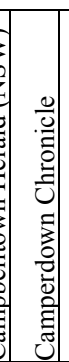 & $\begin{array}{c} \\
\\
0 \\
0 \\
⿹ \\
0 \\
0 \\
0 \\
0 \\
0 \\
0 \\
0 \\
0 \\
0\end{array}$ & 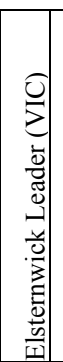 & $\begin{array}{c}0 \\
0 \\
0 \\
0 \\
0 \\
0 \\
0 \\
\frac{3}{0} \\
0 \\
0 \\
0\end{array}$ & 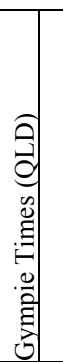 & 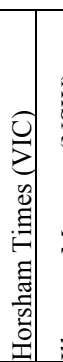 & 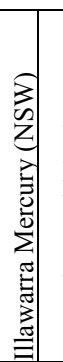 & 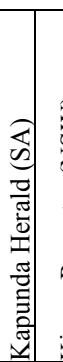 & $\begin{array}{l}3 \\
0 \\
0 \\
0 \\
0 \\
0 \\
0 \\
0 \\
0 \\
0 \\
0 \\
0\end{array}$ & 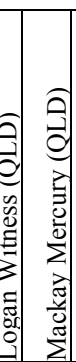 & 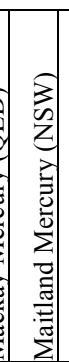 & 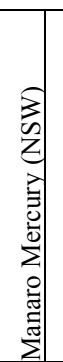 & 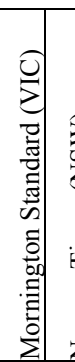 & 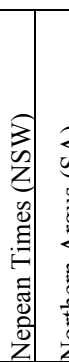 & & & 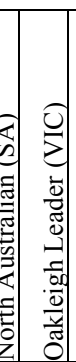 & 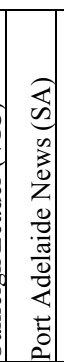 & 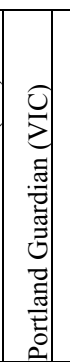 & 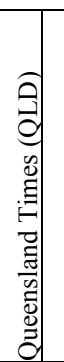 & 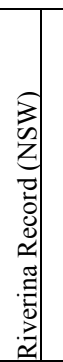 & 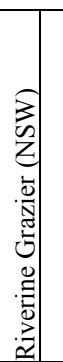 & 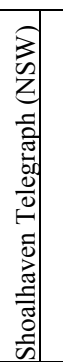 & 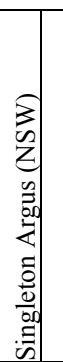 & 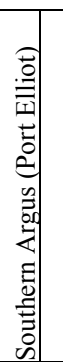 & 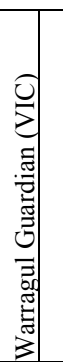 & $\begin{array}{l} \\
0 \\
0 \\
0 \\
0 \\
0 \\
0 \\
0 \\
0 \\
0 \\
0 \\
0 \\
0 \\
3 \\
3\end{array}$ & 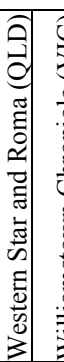 \\
\hline "A Modern Mephistopheles" (1877/8) & Louisa May Alcott & $\mathrm{Am}$ & & & & & $\mathrm{X}$ & & & & & & & & & & & & & & & & & $x$ & & & & & & & & & $\mathrm{X}$ & & & & \\
\hline "A Skeleton in the Closet" (1878/9) & $\begin{array}{l}\text { Mrs, E. D. E. N. } \\
\text { Southworth }\end{array}$ & $\mathrm{Am}$ & & & & & $\mathrm{X}$ & & & & & & & $\mathrm{X}$ & & & & & & & $\mathrm{X}$ & & & $\bar{X}$ & & & & & $\mathrm{X}$ & & $\mathrm{X}$ & & $\mathrm{X}$ & $\mathrm{X}$ & & & \\
\hline "He Wins Who Waits" (1878/9) & Grosvenor Bunster & Aust & & & & & $\mathrm{X}$ & & & & & & & $\mathrm{X}$ & & & & & & & $\mathrm{X}$ & & & $\bar{x}$ & $\mathrm{X}$ & & & & $\mathrm{X}$ & & $\mathrm{X}$ & & $\mathrm{X}$ & & & & \\
\hline "The Bride of Barcelona" (1878/9) & William H. Peck & $\mathrm{Am}$ & & & & & $\mathrm{X}$ & & & & & & & $\mathrm{X}$ & & & & & & & $\mathrm{X}$ & & & $x$ & $\mathrm{X}$ & & & & $\mathrm{X}$ & & $\mathrm{X}$ & & $\mathrm{X}$ & & & & \\
\hline $\begin{array}{l}\text { "Denis Devine, the Australian Gil Blas" } \\
(1880)\end{array}$ & Grosvenor Bunster & Aust & & & & & $\mathrm{X}$ & & & & & & & $\mathrm{X}$ & & & & & & & $\mathrm{X}$ & & & $\bar{x}$ & & & & & & & $\mathrm{X}$ & $\mathrm{X}$ & $\mathrm{X}$ & & & & \\
\hline "The Haunted Grange" (1880) & Mrs Chad & Aust & & & & & $\mathrm{X}$ & & & & & & & $\mathrm{X}$ & & & & & & & $\mathrm{X}$ & & & $x$ & & & $\mathrm{X}$ & & & & $\mathrm{X}$ & $\mathrm{X}$ & $\mathrm{X}$ & & & & \\
\hline "In the Folds of the Serpent" (1880/1) & Donald Cameron & Aust & & & & & $\mathrm{X}$ & & & & & & & $\mathrm{X}$ & & $\mathrm{X}$ & & & & & $\mathrm{X}$ & & & $\bar{x}$ & & & $\mathrm{X}$ & $\mathrm{X}$ & & & $\mathrm{X}$ & $\mathrm{X}$ & $\mathrm{X}$ & & & & \\
\hline "Married to an Actress" $(1880 / 1)$ & Eliza Winstanley & Aust & & & & & $\mathrm{X}$ & & & & & & & & & & & & & & $\mathrm{X}$ & & & $\bar{x}$ & & & & & & & $\mathrm{X}$ & & $\mathrm{X}$ & $\mathrm{X}$ & & & \\
\hline "A Love Race" (1881) & $\begin{array}{l}\text { Robert P. } \\
\text { Whitworth }\end{array}$ & Aust & & & & & $\mathrm{X}$ & & & & & & & $\mathrm{X}$ & & & & & & & $\mathrm{X}$ & & & & & & & & & & $\mathrm{X}$ & $\mathrm{X}$ & $\mathrm{X}$ & & & & \\
\hline “An Unknown Settlement” (1881) & $\begin{array}{l}\text { Robert P. } \\
\text { Whitworth }\end{array}$ & Aust & & & & & $\mathrm{X}$ & & & & & & & $\mathrm{X}$ & & & & & & & $\mathrm{X}$ & & & $\mathrm{X}$ & & & & & & & $\mathrm{X}$ & $\mathrm{X}$ & $\mathrm{X}$ & & & & \\
\hline "Partners" (1881) & Frank Morley & Aust & & & & & $\mathrm{X}$ & & $\mathrm{X}$ & & & & & $\mathrm{X}$ & & & & & & & $\mathrm{X}$ & & & $\mathrm{X}$ & & & & & & & $\mathrm{X}$ & $\mathrm{X}$ & $\mathrm{X}$ & $\mathrm{X}$ & & & \\
\hline "The Ferryman's Secret" (1881) & Sylvanus Cobb & $\mathrm{Am}$ & & & & & $\mathrm{X}$ & & & & & & & $\mathrm{X}$ & & & & & & & $\mathrm{X}$ & & & \begin{tabular}{l|l}
$\mathrm{X}$ & $\mathrm{X}$ \\
$\mathrm{n}$
\end{tabular} & & & & & & & $\mathrm{X}$ & $\mathrm{X}$ & $\mathrm{X}$ & & & & \\
\hline "The Mystery of Major Molineux" (1881) & Marcus Clarke & Aust & & & & & $\mathrm{X}$ & & $\mathrm{X}$ & & & & & $\mathrm{X}$ & & & & & & & $\mathrm{X}$ & & & $\mathrm{x}$ & & & & & & & $\mathrm{X}$ & $\mathrm{X}$ & $\mathrm{X}$ & $\mathrm{X}$ & & & \\
\hline $\begin{array}{l}\text { "Bella's Hero; or The Marquis and the } \\
\text { Freebooter" (1881/2) }\end{array}$ & Sylvanus Cobb & $\mathrm{Am}$ & & & \begin{tabular}{l|l}
$x$ & 2 \\
\end{tabular} & $\mathrm{X}$ & $\mathrm{X}$ & & \begin{tabular}{|l|l|}
$\mathrm{X}$ & \\
\end{tabular} & & & & & 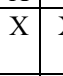 & $\mathrm{X}$ & $\mathrm{X}$ & & & & & $\mathrm{X}$ & & & $\mathrm{X}$ & & & & & $\mathrm{X}$ & & $\mathrm{X}$ & $\mathrm{X}$ & $\mathrm{X}$ & & & & \\
\hline "By Slow Degrees" (1882) & $\begin{array}{l}\text { Alexander } \\
\text { Montgomery } \\
\end{array}$ & Aust & & & & $\mathrm{X}$ & $\mathrm{X}$ & & $\mathrm{X}$ & & $\mathrm{X}$ & & & $\mathrm{X}$ & $\mathrm{X}$ & & & & & & & & & $\mathrm{X}$ & & & & & $\mathrm{X}$ & & $\mathrm{X}$ & $\mathrm{X}$ & $\mathrm{X}$ & & & & \\
\hline "Grit" (1882) & Donald Cameron & Aust & & & & $\mathrm{T}$ & $\mathrm{X}$ & & $\mathrm{X}$ & & & & & $\mathrm{X}$ & $\mathrm{X}$ & & & & & & $\mathrm{T}$ & & & $\mathrm{X}$ & & & & & & & $\mathrm{T}$ & $\mathrm{T}$ & $\mathrm{X}$ & & & & \\
\hline "Saved by a Ring" (1882/3) & Harold W. H. & Aust & & & & \begin{tabular}{l|l}
$\mathrm{X}$ & $\mathrm{z}$ \\
$\mathrm{n}$
\end{tabular} & $\mathrm{X}$ & & $\mathrm{X}$ & & $\mathrm{X}$ & & & $\mathrm{X}$ & & & & & & & $\mathrm{X}$ & & & $\mathrm{X}$ & & & & & $X$ & & \begin{tabular}{l|l}
$\mathrm{X}$ \\
\end{tabular} & $\mathrm{X}$ & & & & \begin{tabular}{l|l}
$\mathrm{X}$ \\
\end{tabular} & \\
\hline
\end{tabular}




\begin{tabular}{|c|c|c|c|c|c|c|c|c|c|c|c|c|c|c|c|c|c|c|c|c|c|c|c|c|c|c|c|c|c|c|c|c|c|}
\hline & Stephen & & & & & & & & & & & & & & & & & & & & & & & & & & & & & & & & \\
\hline "Theodora Vail" (1883) & Janet Carroll & Aust & & & $\mathrm{X}$ & $\mathrm{X}$ & $\mathrm{X}$ & $\mathrm{X}$ & & & $\mathrm{T}$ & $\mathrm{X}$ & & & & & & $\mathrm{X}$ & & & & & & & & $\mathrm{X}$ & $\mathrm{X}$ & & & & & & \\
\hline "Ettie’s Error" (1883) & $\begin{array}{l}\text { Harold W. H. } \\
\text { Stephen }\end{array}$ & Aust & & $\mathrm{T}$ & $\mathrm{X}$ & $\mathrm{X}$ & $\mathrm{X}$ & $\frac{1}{x}$ & $\mathrm{X}$ & & $\mathrm{X}$ & $\mathrm{X}$ & & & $\mathrm{X}$ & $\mathrm{X}$ & & & & & & & & & & $\mathrm{X}$ & $\mathrm{X}$ & $\mathrm{X}$ & & & $\mathrm{X}$ & & \\
\hline "Evangeline" (1883) & Donald Cameron & Aust & & $\mathrm{T}$ & $\mathrm{X}$ & $\mathrm{X}$ & $\mathrm{X}$ & $\mathrm{x}$ & $\mathrm{X}$ & & & & & & $\mathrm{T}$ & & & & & & & & & & & & $\mathrm{T}$ & & & & & & $\mathrm{T}$ \\
\hline $\begin{array}{l}\text { "Harry Bloomfield; or, The Adventures of } \\
\text { an Early Australian Squatter" (1883) }\end{array}$ & Angus McLean & Aust & & $\mathrm{X}$ & $\mathrm{X}$ & $\mathrm{X}$ & $\mathrm{X}$ & $\mathrm{X}$ & $\mathrm{X}$ & & $\mathrm{X}$ & & & & & & & & & & & & & & & & $\mathrm{X}$ & $\mathrm{X}$ & & & $\mathrm{X}$ & & $\mathrm{X}$ \\
\hline "Kitty Dunolly, My Schoolmate" (1883) & \# G. E. C. & Unk & & & $\mathrm{X}$ & $\mathrm{X}$ & $\mathrm{X}$ & $\mathrm{x}$ & $\mathrm{X}$ & & & & & & & $\mathrm{X}$ & & & & & & & & & & $\mathrm{X}$ & $\mathrm{X}$ & $\mathrm{T}$ & & & $\mathrm{X}$ & & \\
\hline “Mrs Birtles’ Boarders" (1883) & $\begin{array}{l}\text { Robert P. } \\
\text { Whitworth }\end{array}$ & Aust & & & $\mathrm{X}$ & $\mathrm{X}$ & $\mathrm{X}$ & $\mathrm{x}$ & $\mathrm{X}$ & & & $\mathrm{T}$ & & & $\mathrm{X}$ & $\mathrm{X}$ & & & & & & & & & & & $\mathrm{X}$ & $\mathrm{X}$ & & & $\mathrm{X}$ & & \\
\hline "Harriette Hurst's Christmas" (1883/4) & Janet Carroll & Aust & & & $\mathrm{X}$ & $\mathrm{X}$ & & & $\mathrm{X}$ & & & & & & & & & & & & & & & & & $\mathrm{x}$ & $\mathrm{X}$ & $\mathrm{X}$ & & & $\mathrm{X}$ & & \\
\hline "Urbain and Isette" (1883/4) & George R. Sims & Brit & & & $\mathrm{X}$ & $\mathrm{X}$ & & & $\mathrm{X}$ & & $\mathrm{X}$ & & & & & $\mathrm{X}$ & & & $\mathrm{X}$ & & & $\mathrm{X}$ & & $\mathrm{X}$ & & $\mathrm{X}$ & $\mathrm{X}$ & $\mathrm{X}$ & & & & & \\
\hline $\begin{array}{l}\text { "A Witness for the Defence" } \\
(1884)\end{array}$ & R. E. Matthews & Unk & & & $\mathrm{X}$ & $\mathrm{X}$ & $\mathrm{X}$ & $\mathrm{x}$ & $\mathrm{X}$ & & $\mathrm{X}$ & $\mathrm{X}$ & $\mathrm{X}$ & & & $\mathrm{X}$ & & & & & & $\mathrm{X}$ & & $\mathrm{X}$ & & $\mathrm{X}$ & $\mathrm{X}$ & $\mathrm{X}$ & & & & $\mathrm{X}$ & \\
\hline "Alice" (1884) & Donald Cameron & Aust & & & $\mathrm{X}$ & $\mathrm{X}$ & & & $\mathrm{X}$ & & $\mathrm{X}$ & & $\mathrm{X}$ & & $\mathrm{X}$ & & & & & & & $\mathrm{X}$ & & $\mathrm{X}$ & & $\mathrm{X}$ & $\mathrm{X}$ & $\mathrm{X}$ & & & & & \\
\hline "Bonshaw" (1884) & \# Magnus Badge & Unk & & & $\mathrm{X}$ & $\mathrm{X}$ & & & & & $\mathrm{X}$ & & $\mathrm{X}$ & & & $\mathrm{X}$ & & & & & & $\mathrm{X}$ & & & & $\mathrm{X}$ & $\mathrm{X}$ & $\mathrm{X}$ & & & $\mathrm{X}$ & & \\
\hline "Dan Lyons' Doom" (1884) & Waif Wander & Aust & & & $\mathrm{X}$ & $\mathrm{X}$ & & & $\mathrm{X}$ & & $\mathrm{X}$ & $\mathrm{X}$ & $\mathrm{X}$ & & & $\mathrm{X}$ & & & & & & $\mathrm{X}$ & & $\mathrm{T}$ & & $\mathrm{X}$ & $\mathrm{X}$ & $\mathrm{X}$ & & & & & \\
\hline "Intrigue" (1884) & $\begin{array}{l}\text { Brigadier General } \\
\text { Henry Ronald } \\
\text { McIver }\end{array}$ & $\mathrm{Am}$ & & & $\mathrm{X}$ & $\mathrm{X}$ & & & $\mathrm{X}$ & & $\mathrm{X}$ & & $\mathrm{X}$ & & & $\mathrm{X}$ & & & & & & $\mathrm{X}$ & & $\mathrm{X}$ & & $\mathrm{X}$ & $\mathrm{X}$ & $\mathrm{X}$ & & & $\mathrm{X}$ & & \\
\hline $\begin{array}{l}\text { "Lindigo the White Woman; or, The } \\
\text { Highland Girl's Captivity among } \\
\text { Australian Blacks" (1884) }\end{array}$ & Angus McLean & Aust & & & $\mathrm{X}$ & $\mathrm{X}$ & & & $\mathrm{X}$ & & $\mathrm{X}$ & $\mathrm{X}$ & $\mathrm{X}$ & & & $\mathrm{X}$ & & & & & & $\mathrm{X}$ & & $\mathrm{X}$ & & $\mathrm{X}$ & $\mathrm{X}$ & $\mathrm{X}$ & & & $\mathrm{X}$ & & \\
\hline "Marc Grecli" (1884) & Janet Carroll & Aust & & & $\mathrm{X}$ & $\mathrm{X}$ & & & $\mathrm{X}$ & & $\mathrm{X}$ & & $\mathrm{X}$ & & & $\mathrm{X}$ & & & & & & $\mathrm{X}$ & & $\mathrm{X}$ & & $\mathrm{X}$ & $\mathrm{X}$ & $\mathrm{X}$ & & & $\mathrm{X}$ & & \\
\hline "Philip Penfold" (1884) & Frank Morley & Aust & & & $x$ & $\mathrm{X}$ & & & $\mathrm{X}$ & & $\mathrm{X}$ & & $\mathrm{X}$ & & & & & & & & & $\mathrm{X}$ & & $x$ & & $\mathrm{x}$ & $\mathrm{X}$ & $\mathrm{X}$ & & & $\begin{array}{l}x \\
\end{array}$ & $\mathrm{~T}$ & \\
\hline “A Terrible Legacy" (1884/5) & Grosvenor Bunster & Aust & & & $\mathrm{X}$ & $\mathrm{X}$ & & & $\mathrm{X}$ & & $\mathrm{X}$ & $\mathrm{X}$ & $\mathrm{X}$ & & $\mathrm{X}$ & $\mathrm{X}$ & & & & & & $\mathrm{X}$ & & $\mathrm{X}$ & & $\mathrm{X}$ & $\mathrm{X}$ & $\mathrm{X}$ & & & $\mathrm{X}$ & & \\
\hline “After Many Days" (1884/5) & $\begin{array}{l}\text { Robert P. } \\
\text { Whitworth }\end{array}$ & Aust & & & $\mathrm{X}$ & $\mathrm{X}$ & & & $\mathrm{X}$ & & $\mathrm{X}$ & $\mathrm{X}$ & $\mathrm{X}$ & & $\mathrm{X}$ & & & & & & & $\mathrm{X}$ & & $\mathrm{X}$ & & $\mathrm{X}$ & $\mathrm{X}$ & $\mathrm{X}$ & & & $\mathrm{X}$ & & \\
\hline "An Australian Pilgrimage" (1884/5) & Lee L'Acton & Unk & & & $\mathrm{X}$ & $\mathrm{X}$ & & & $\mathrm{X}$ & & $\mathrm{X}$ & $\mathrm{X}$ & $\mathrm{X}$ & & $\mathrm{X}$ & $\mathrm{X}$ & & & & & & $\mathrm{X}$ & & $\mathrm{X}$ & & $\mathrm{X}$ & $\mathrm{X}$ & $\mathrm{X}$ & & & $\mathrm{X}$ & & \\
\hline "Orinora, A Pacific Idyll" (1884/5) & Donald Cameron & Aust & & & $\mathrm{X}$ & $\mathrm{X}$ & & & $\mathrm{X}$ & & $\mathrm{X}$ & $\mathrm{X}$ & $\mathrm{X}$ & & & & & & & & & $\mathrm{X}$ & & $\mathrm{X}$ & & $\mathrm{X}$ & $\mathrm{X}$ & $\mathrm{X}$ & & & & & \\
\hline "An Apostle of the Tules" (1885) & Bret Harte & $\mathrm{Am}$ & & & $\mathrm{X}$ & & & & & & $\mathrm{X}$ & & & & & & $\mathrm{X}$ & $\mathrm{x}$ & & & & & $\mathrm{x}$ & & & & & $\mathrm{X}$ & & & $\mathrm{X}$ & & \\
\hline "Darkest Before Dawn" (1885) & $\begin{array}{l}\text { Robert P. } \\
\text { Whitworth }\end{array}$ & Aust & & & $\mathrm{X}$ & $\mathrm{X}$ & & & & & $\mathrm{T}$ & & $\mathrm{X}$ & & & & & & & & & $\mathrm{X}$ & & & & $\mathrm{T}$ & $\mathrm{T}$ & $\mathrm{X}$ & & & & & \\
\hline "His One Mistake" (1885) & Bertha M. Clay & $\mathrm{Am}$ & & & $\mathrm{X}$ & $\mathrm{X}$ & & & $\mathrm{X}$ & & $\mathrm{X}$ & $\mathrm{X}$ & $\mathrm{X}$ & & & $\mathrm{X}$ & & & & & & $\mathrm{X}$ & & $\mathrm{X}$ & & $\mathrm{X}$ & $\mathrm{X}$ & $\mathrm{X}$ & & & $\mathrm{X}$ & & \\
\hline "Little Count Tista" (1885) & Rita & Brit & & & $\mathrm{X}$ & & & & & & & $\mathrm{X}$ & & & & & & & & & & & & & & $\mathrm{T}$ & & & & & & & \\
\hline "Poor Little Life" (1885) & Sheriff Rampini & Brit & & & & $\mathrm{T}$ & & & $\mathrm{X}$ & & $\mathrm{X}$ & & $\mathrm{T}$ & & & & & & & & & $\mathrm{X}$ & & $\mathrm{T}$ & & $\mathrm{T}$ & & & & & & & \\
\hline "Royal Love" (1885) & Wilkie Collins & Brit & & & $\mathrm{X}$ & $\mathrm{X}$ & & & & & $\mathrm{X}$ & & $\mathrm{X}$ & & & $\mathrm{X}$ & & & & & & $\mathrm{X}$ & & & & & $\mathrm{X}$ & $\mathrm{X}$ & & & $\mathrm{X}$ & & \\
\hline "Yvonne" (1885) & Unknown & Unk & & & $\mathrm{T}$ & $\mathrm{X}$ & & & & & $\mathrm{X}$ & & $\mathrm{X}$ & & & & & & & & & $\mathrm{X}$ & & & & $\mathrm{T}$ & $\mathrm{X}$ & $\mathrm{X}$ & & & & & \\
\hline $\begin{array}{l}\text { "Put Asunder; Or Lady Castlemaine's } \\
\text { Divorce" (1885/6) }\end{array}$ & Charlotte Brame & Brit & & & $\mathrm{X}$ & $\mathrm{X}$ & & & $\mathrm{X}$ & & $\mathrm{T}$ & $\mathrm{X}$ & $\mathrm{X}$ & & $\mathrm{X}$ & $\mathrm{X}$ & & & & $\mathrm{X}$ & & $\mathrm{X}$ & & $\mathrm{X}$ & & $\mathrm{X}$ & $\mathrm{X}$ & $\mathrm{X}$ & & & & & \\
\hline $\begin{array}{l}\text { "Carmeline; Or, The Convict's Bride" } \\
(1886)\end{array}$ & $\begin{array}{l}\text { Francis A. } \\
\text { Durivage }\end{array}$ & $\mathrm{Am}$ & & & $\mathrm{X}$ & $\mathrm{X}$ & & & & $\mathrm{X}$ & 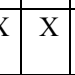 & $\mathrm{X}$ & $\mathrm{X}$ & & $\mathrm{X}$ & & & & & & & $\mathrm{X}$ & & & & $\mathrm{X}$ & $\mathrm{X}$ & $\mathrm{X}$ & & & & & \\
\hline "John Abberton" (1886) & Grosvenor Bunster & Aust & & & $\mathrm{X}$ & $\mathrm{X}$ & & & & & $\mathrm{X}$ & $\mathrm{X}$ & $\mathrm{X}$ & & $\mathrm{X}$ & $\mathrm{T}$ & & & & & & $\mathrm{X}$ & & $\mathrm{X} \mid$ & & 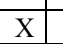 & $\mathrm{X}$ & $\mathrm{X}$ & & & & & \\
\hline “A Race for Life” (1886/7) & $\begin{array}{l}\text { Robert P. } \\
\text { Whitworth }\end{array}$ & Aust & & & $\mathrm{X}$ & $\mathrm{X}$ & & & & & $\mathrm{X}$ & & $\mathrm{X}$ & & $\mathrm{X}$ & & $\mathrm{X}$ & & & & & $\mathrm{X}$ & & $\mathrm{X}$ & $\mathrm{X}$ & & & $\mathrm{X}$ & & & & & \\
\hline "Alma" (1886/7) & $\begin{array}{l}\text { Harold W. H. } \\
\text { Stephen }\end{array}$ & Aust & & & $\mathrm{X}$ & $\mathrm{X}$ & & & & & $\mathrm{X}$ & $\mathrm{X}$ & $\mathrm{X}$ & & $\mathrm{X}$ & & $\mathrm{X}$ & & & & & $\mathrm{X}$ & & $\mathrm{X}$ & $\mathrm{x}$ & $\mathrm{x}$ & $\mathrm{X}$ & $\mathrm{X}$ & & & & & \\
\hline "Dora Dunbar" (1886/7) & Mona & Aust & & & $\mathrm{X}$ & $\mathrm{X}$ & & & & & & & $\mathrm{X}$ & & $\mathrm{X}$ & & $\mathrm{X}$ & & & $\mathrm{X}$ & & $\mathrm{X}$ & & $\mathrm{X}$ & & $\mathrm{X}$ & $\mathrm{X}$ & $\mathrm{X}$ & & & & & \\
\hline "Fifty Lashes" (1887) & Atha Westbury & Aust & & & $\mathrm{X}$ & $\mathrm{X}$ & & & & & $\mathrm{X}$ & & $\mathrm{X}$ & & $\mathrm{X}$ & & $\mathrm{X}$ & & & & & $\mathrm{X}$ & & & $\mathrm{X}$ & & & $\mathrm{X}$ & & & & & \\
\hline "Hawksnest" (1887) & Atha Westbury & Aust & & & & $\mathrm{X}$ & & & & & $\mathrm{X}$ & & $\mathrm{X}$ & & & & $\mathrm{X}$ & & & & & & & & $\mathrm{X}$ & & & $\mathrm{X}$ & & & & & \\
\hline $\begin{array}{l}\text { "Days of Crime and Years of Suffering" } \\
(1887 / 8)\end{array}$ & Owen Suffolk & Aust & & & & $\mathrm{X}$ & & & & $\mathrm{X}$ & $\mathrm{X}$ & $\mathrm{X}$ & $\mathrm{X}$ & & $\mathrm{X}$ & & $\mathrm{X}$ & & & & $\mathrm{X}$ & $\mathrm{X}$ & & $\mathrm{X}$ & $\mathrm{X}$ & $\mathrm{X}$ & & $\mathrm{X}$ & & & & & \\
\hline $\begin{array}{l}\text { "Florabel's Lover; Or, Rival Belles" } \\
\text { (published as "The Rival Belles of } \\
\text { Parramatta") (1888) }\end{array}$ & Laura Jean Libbey & $\mathrm{Am}$ & & & & $\mathrm{X}$ & & & & & & $\mathrm{X}$ & $\mathrm{X}$ & & $\mathrm{X}$ & & $\mathrm{X}$ & & & & & & & \begin{tabular}{l|l}
$x$ \\
\end{tabular} & $\mathrm{X}$ & $\mathrm{X}$ & & $\mathrm{X}$ & & & & & \\
\hline $\begin{array}{l}\text { "Hunted Down; Or, The Clink of Gold" } \\
\text { (1888) }\end{array}$ & Edmund Bury & Unk & & & & $\mathrm{X}$ & & & & & & $\mathrm{X}$ & $\mathrm{X}$ & & $\mathrm{X}$ & & $\mathrm{X}$ & & & & & & & & & $\mathrm{X}$ & & $\mathrm{X}$ & & & & & \\
\hline
\end{tabular}




\begin{tabular}{|c|c|c|c|c|c|c|c|c|c|c|c|c|c|c|c|c|c|c|c|c|c|c|c|c|c|c|c|c|c|c|}
\hline $\begin{array}{l}\text { "The False Knight; Or, The Robber of the } \\
\text { Black Forest" (1888) }\end{array}$ & Sylvanus Cobb & $\mathrm{Am}$ & & & & & $\mathrm{X}$ & & & & & $\mathrm{X}$ & $\mathrm{X}$ & $\mathrm{X}$ & & & $\mathrm{X}$ & & & $\mathrm{X}$ & & & & $\mathrm{X}$ & $\mathrm{X}$ & $\mathrm{X}$ & $\mathrm{X}$ & & & \\
\hline $\begin{array}{l}\text { "The Midnight Prophecy; Or, Heir of } \\
\text { Strathspey Towers" (1888/9) }\end{array}$ & $\begin{array}{l}\text { Emma Garrison } \\
\text { Jones }\end{array}$ & $\mathrm{Am}$ & $\mathrm{X}$ & & & & $\mathrm{T}$ & & & & & $\mathrm{X}$ & $\mathrm{X}$ & $\mathrm{X}$ & & $\mathrm{X}$ & $\mathrm{X}$ & & & $\mathrm{X}$ & & & & $\mathrm{T}$ & $\mathrm{X}$ & $\mathrm{X}$ & $\mathrm{X}$ & $\mathrm{X}$ & & \\
\hline $\begin{array}{l}\text { "Caged; Or, Mrs Marchmont’s Marriage" } \\
(1889)\end{array}$ & L. I. N. & Unk & & & & & $\mathrm{T}$ & & & & & & $\mathrm{X}$ & $\mathrm{X}$ & & & $\mathrm{X}$ & & & $\mathrm{X}$ & & & & & $\mathrm{X}$ & $\mathrm{X}$ & $\mathrm{X}$ & & & \\
\hline $\begin{array}{l}\text { "Fairly Caught; Or, He Would Marry } \\
\text { Money"(1889) }\end{array}$ & Annie G. Hopkins & Brit & & & & & $\mathrm{T}$ & & & & & & $\mathrm{T}$ & $\mathrm{X}$ & & & $\mathrm{T}$ & & & $\mathrm{X}$ & & & & & $\mathrm{T}$ & $\mathrm{X}$ & $\mathrm{T}$ & & & \\
\hline $\begin{array}{l}\text { "The Secret Chamber; Or, The Gipsy's } \\
\text { Revenge" (1889/90) }\end{array}$ & Eliza A. Dupuy & $\mathrm{Am}$ & & & & & $\mathrm{X}$ & & & & & $\mathrm{X}$ & $\mathrm{X}$ & $\mathrm{X}$ & $\mathrm{X}$ & $\mathrm{X}$ & $\mathrm{X}$ & & & $\mathrm{X}$ & $\mathrm{X}$ & & & $\mathrm{X}$ & $\mathrm{X}$ & $\mathrm{X}$ & $\mathrm{X}$ & $\mathrm{X}$ & & \\
\hline $\begin{array}{l}\text { "Ruby's Marriage; Or, The Tragedy of a } \\
\text { Life" (1890) }\end{array}$ & G. A. W. & Unk & & & & & $\mathrm{X}$ & & & & & $\mathrm{X}$ & $\mathrm{X}$ & $\mathrm{X}$ & & $\mathrm{X}$ & $\mathrm{X}$ & & & $\mathrm{X}$ & & & & $\mathrm{X}$ & $\mathrm{X}$ & $\mathrm{X}$ & $\mathrm{X}$ & $\mathrm{X}$ & & \\
\hline "A Life at Stake" (1890/1) & Harriet Lewis & $\mathrm{Am}$ & & $\mathrm{X}$ & & & $\mathrm{X}$ & & & & & $\mathrm{X}$ & $\mathrm{X}$ & $\mathrm{X}$ & & $\mathrm{X}$ & & & & & $\mathrm{X}$ & & & & $\mathrm{X}$ & $\mathrm{X}$ & $\mathrm{X}$ & $\mathrm{X}$ & & \\
\hline $\begin{array}{l}\text { "Nadja; Or, A Battle With Death" } \\
(1890 / 1)\end{array}$ & Frederick M. Dey & $\mathrm{Am}$ & & & & & $\mathrm{X}$ & & & $\mathrm{T}$ & & $\mathrm{X}$ & $\mathrm{X}$ & $\mathrm{X}$ & & $\mathrm{X}$ & $\mathrm{X}$ & & & & $\mathrm{X}$ & & & $\mathrm{T}$ & $\mathrm{x}$ & $\mathrm{X}$ & $\mathrm{X}$ & $\mathrm{X}$ & & \\
\hline $\begin{array}{l}\text { "The Haunted Husband; Or, Lady } \\
\text { Chetwynd's Spectre" (1890/1) }\end{array}$ & Harriet Lewis & $\mathrm{Am}$ & & & & & $\mathrm{X}$ & $\mathrm{X}$ & & & & $\mathrm{X}$ & $\mathrm{X}$ & $\mathrm{X}$ & & $\mathrm{X}$ & $\mathrm{X}$ & & & $\mathrm{X}$ & $\mathrm{X}$ & & & $\mathrm{X}$ & $\mathrm{X}$ & $\mathrm{X}$ & $\mathrm{X}$ & $\mathrm{X}$ & & \\
\hline "Jephthah's Daughter" (1891) & Julia Magruder & $\mathrm{Am}$ & & $\mathrm{X}$ & & & $\mathrm{X}$ & & & & & $\mathrm{T}$ & $\mathrm{X}$ & $\mathrm{X}$ & & & & & & & $\mathrm{X}$ & & & $\mathrm{X}$ & $\mathrm{X}$ & $\mathrm{X}$ & $\mathrm{X}$ & & & \\
\hline $\begin{array}{l}\text { "Ottilie Aster's Silence" (published as } \\
\text { "The Eventful Secret") (1891) }\end{array}$ & Ida Boy-Ed & Ger & & $\mathrm{X}$ & & & $\mathrm{X}$ & & & $\mathrm{X}$ & & $\mathrm{T}$ & $\mathrm{X}$ & & & & & $\mathrm{X}$ & & & $\mathrm{X}$ & & & $\mathrm{X}$ & $\mathrm{X}$ & $\mathrm{X}$ & $\mathrm{X}$ & $\mathrm{X}$ & & \\
\hline "The Mount Macedon Mystery" (1891) & James J. Wright & Aust & & $\mathrm{X}$ & & & $\mathrm{X}$ & & & $\mathrm{X}$ & & $\mathrm{X}$ & $\mathrm{X}$ & & & & & & & & $\mathrm{X}$ & & & $\mathrm{X}$ & $\mathrm{X}$ & $\mathrm{X}$ & $\mathrm{X}$ & $\mathrm{X}$ & & \\
\hline "The Verdict Was --?” (1891) & $\begin{array}{l}\text { Harold M. } \\
\text { Mackenzie }\end{array}$ & Aust & & $\mathrm{X}$ & & & $\mathrm{X}$ & & & & & & $\mathrm{X}$ & $\mathrm{X}$ & & & & & & & $\mathrm{X}$ & & & & $\mathrm{X}$ & $\mathrm{X}$ & $\mathrm{X}$ & $\mathrm{X}$ & & \\
\hline "Fifty Lashes" (1891/2) & Atha Westbury & Aust & & & & & & $\mathrm{X}$ & & & & & & $\mathrm{X}$ & & & & & & & & & & & $\mathrm{X}$ & & $\mathrm{X}$ & $\mathrm{X}$ & $\mathrm{X}$ & \\
\hline "The Bottle Imp" (1891/2) & $\begin{array}{l}\text { Robert Lewis } \\
\text { Stevenson } \\
\end{array}$ & Brit & & & & & $\mathrm{X}$ & & & & & $\mathrm{T}$ & $\mathrm{X}$ & $\mathrm{X}$ & & & & & & & $\mathrm{X}$ & & & & $\mathrm{X}$ & $\mathrm{X}$ & $\mathrm{X}$ & & & \\
\hline "A Dead Letter" (1892) & Unknown & Unk & & $\mathrm{X}$ & & & & $\mathrm{X}$ & & & & $\mathrm{T}$ & & $\mathrm{X}$ & & & & & & & & & & & $\mathrm{X}$ & & $\mathrm{X}$ & & $\mathrm{X}$ & \\
\hline $\begin{array}{l}\text { "The Shadow of Hilton Fernbrook" } \\
\text { (1892) }\end{array}$ & Atha Westbury & Aust & & $\mathrm{X}$ & & & $\mathrm{X}$ & $\mathrm{X}$ & & & & $\mathrm{X}$ & & $\mathrm{X}$ & & & & & $\mathrm{X}$ & & $\mathrm{X}$ & & & & $\mathrm{X}$ & & $\mathrm{X}$ & & $\mathrm{X}$ & \\
\hline "Suhrab, the Turkoman Hero" (1892) & Ferdinand Braun & Unk & & $\mathrm{X}$ & & & & $\mathrm{X}$ & & & & $\mathrm{X}$ & & $\mathrm{X}$ & & & & & & & $\mathrm{T}$ & & & & $\mathrm{X}$ & & $\mathrm{X}$ & & $\mathrm{X}$ & \\
\hline
\end{tabular}

\section{Syndicate 2}

\begin{tabular}{|c|c|c|c|c|c|c|c|c|c|c|c|c|c|c|c|c|c|c|c|c|}
\hline Title & Author & Nat. & 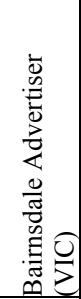 & 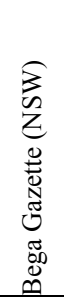 & 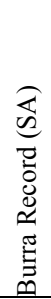 & 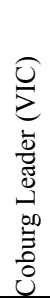 & 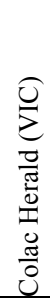 & 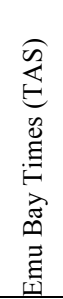 & $\begin{array}{l}0 \\
0 \\
\sum \\
0 \\
0 \\
0 \\
0 \\
0 \\
0 \\
5 \\
0 \\
0 \\
0\end{array}$ & 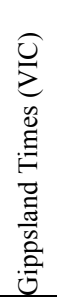 & 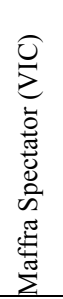 & 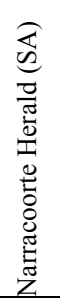 & 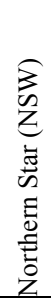 & 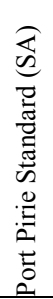 & 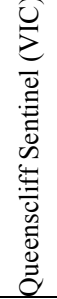 & 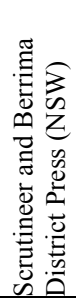 & 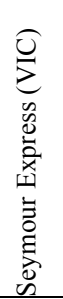 & 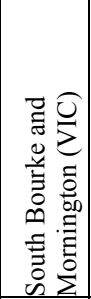 & 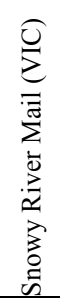 & 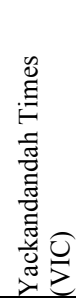 \\
\hline "Mrs Lorrimer's Advertisement" (1883) & \# Bert Ferres & Unk & & $\bar{x}$ & & & & & & & & & & & & & & $\mathrm{X}$ & & \\
\hline "The Bread of the Angels" (1883) & E. M. Verano & Ger & & $\mathrm{X}$ & & & & & & & & & & & & & & $\mathrm{X}$ & & \\
\hline "Dark Days" (1884) & George Manville Fenn & Brit & & $\mathrm{X}$ & & & & & & & & & $\mathrm{X}$ & & $\mathrm{X}$ & & & $\mathrm{X}$ & & \\
\hline "The Bride of a Day" (1884) & Charlotte Despard & Brit & & $\mathrm{X}$ & & & & & & & & & $\mathrm{X}$ & & $\mathrm{X}$ & & & $\mathrm{X}$ & & \\
\hline "The Picture" (1884) & Charles Reade & Brit & & $\mathrm{X}$ & & & & & & $\mathrm{X}$ & & & & & $\mathrm{X}$ & & & $\mathrm{X}$ & & \\
\hline "Mr Pudster's Return" (1884/5) & W. Laird Clowes & Brit & & $\mathrm{X}$ & & & $\mathrm{X}$ & & & $\mathrm{X}$ & & & & & $\mathrm{X}$ & & & $\mathrm{X}$ & & \\
\hline "Revised History of England" (1885) & The Baron & Unk & $\mathrm{X}$ & $\mathrm{X}$ & & & $\mathrm{X}$ & & & & & & & & $\mathrm{X}$ & & & $\mathrm{X}$ & & \\
\hline "Sylvester's Wife" (1885) & Ernest Dodson & Unk & $\mathrm{X}$ & $\mathrm{X}$ & & & $\mathrm{X}$ & & & & & & & & $\mathrm{X}$ & & & $\mathrm{X}$ & & \\
\hline "Terror-stricken" (1885) & $\begin{array}{l}\text { Themselves and Some } \\
\text { Others }\end{array}$ & Unk & & $\mathrm{x}$ & & & $\mathrm{X}$ & & & & & & & & & & & $\mathrm{X}$ & & \\
\hline "The Miner's Partner" (1885) & Henry Tinson & Brit & & $\mathrm{X}$ & & & $\mathrm{X}$ & & & & & & & & $\mathrm{X}$ & & & $\mathrm{X}$ & & \\
\hline "A Charity Child" (1885/6) & Unknown & Unk & $\mathrm{X}$ & & & & $\mathrm{X}$ & & & & & & & & & & & $\mathrm{X}$ & & \\
\hline
\end{tabular}




\begin{tabular}{|c|c|c|c|c|c|c|c|c|c|c|c|c|c|c|c|c|c|c|c|}
\hline "The Convict's Double" (1886) & \# J. A. Andrews & Unk & & & & $\mathrm{X}$ & & & & & & & & & & & $\mathrm{X}$ & & \\
\hline "Paraded in Print" (1886) & \# C. B. M. & Unk & $\mathrm{X}$ & & & $\mathrm{X}$ & & & & & & & & & & & $\mathrm{X}$ & & \\
\hline "The Royal Mummy" (1886) & \# V. J. Daley & Unk & & & & $\mathrm{X}$ & & & & & & & & & & & $\mathrm{X}$ & & \\
\hline "The Jullabad Tragedy" (1888) & P. L. McDermott & Unk & & & & & & & & & & & & $\mathrm{X}$ & & $\mathrm{X}$ & $\mathrm{X}$ & & \\
\hline "Through the Furnace" (1888) & Joseph Crawford Scott & Brit & & & & & & & & & & & & $\mathrm{X}$ & & $\mathrm{X}$ & $\mathrm{X}$ & & \\
\hline "With a Show Around the World" (1888) & Edward Andrew Glover & $\mathrm{Am}$ & & & & & & & & & & & & $\mathrm{X}$ & & $\mathrm{X}$ & $\mathrm{X}$ & & \\
\hline "Beauty and the Beasts" (1888/9) & Charles Rhoades & Unk & & & & & & & & & & & & $\mathrm{X}$ & & $\mathrm{X}$ & $\mathrm{X}$ & & \\
\hline "Strayed from the Flock" (1890) & Unknown & Unk & & & & & & & $\mathrm{T}$ & & & & & $\mathrm{X}$ & & & $\mathrm{X}$ & & \\
\hline $\begin{array}{l}\text { "The Manager's Favorite; Or, The } \\
\text { Tragedy of Carnleigh Shores" (1890) }\end{array}$ & Clara Augusta Jones Trask & $\mathrm{Am}$ & $\mathrm{X}$ & & & & & $\mathrm{X}$ & $\mathrm{X}$ & & & $\mathrm{X}$ & & & & & & & \\
\hline "My Lady's Revenge" (1890) & Emma Garrison Jones & $\mathrm{Am}$ & & & $\mathrm{X}$ & & & $\mathrm{X}$ & & $\mathrm{X}$ & $\mathrm{X}$ & & & & & & & & $\mathrm{X}$ \\
\hline "The Crime of a Countess" (1890) & Nick Carter & $\mathrm{Am}$ & & & & & & $\mathrm{X}$ & & $\mathrm{X}$ & & & & & & & & & \\
\hline "The Rajah's Vengeance" (1890) & Frederick M. Dey & $\mathrm{Am}$ & & $\mathrm{X}$ & $\mathrm{X}$ & & & $\mathrm{X}$ & & $\mathrm{X}$ & $\mathrm{X}$ & & & & $\mathrm{X}$ & & & & $\mathrm{X}$ \\
\hline "From Prison to Parliament" (1891) & James J. Wright & Aust & & & $\mathrm{X}$ & & $\mathrm{X}$ & $\mathrm{X}$ & & $\mathrm{X}$ & $X$ & & & $\mathrm{X}$ & $\mathrm{X}$ & & & & $\mathrm{X}$ \\
\hline "In Love's Crucible" (1891) & Bertha M. Clay & $\mathrm{Am}$ & & & $\mathrm{X}$ & & $\mathrm{X}$ & $\mathrm{X}$ & & $\mathrm{X}$ & $\mathrm{X}$ & & & & & & & & $\mathrm{X}$ \\
\hline $\begin{array}{l}\text { "O’Neil McDarragh, the Irish Detective; } \\
\text { Or, The Strategy of a Brave Man" (1891) }\end{array}$ & Harlan P. Halsey & $\mathrm{Am}$ & & & $\mathrm{X}$ & & $\mathrm{X}$ & $\mathrm{X}$ & & $\mathrm{x}$ & $\mathrm{X}$ & & & & & & & & $\mathrm{x}$ \\
\hline $\begin{array}{l}\text { "The American Detective in Russia; Or } \\
\text { Piping a Conspiracy" (published as } \\
\text { "Barnes, the Australian Detective" (1891) }\end{array}$ & Harlan P. Halsey & $\mathrm{Am}$ & & & $\mathrm{X}$ & & $\mathrm{X}$ & $\mathrm{X}$ & & $\mathrm{X}$ & $\mathrm{X}$ & & & $\mathrm{X}$ & & & & & $\mathrm{X}$ \\
\hline "Re-United" (1892) & Unknown & Unk & & & & & $\mathrm{X}$ & $\mathrm{X}$ & & $\mathrm{X}$ & $\mathrm{X}$ & & $\mathrm{X}$ & $\mathrm{X}$ & & & & & $\mathrm{X}$ \\
\hline "Misericordia" (1892/3) & A. G. R. & Unk & & & $\mathrm{X}$ & & $\mathrm{X}$ & & & & $\mathrm{X}$ & & $\mathrm{X}$ & $\mathrm{X}$ & & & & $\mathrm{X}$ & \\
\hline
\end{tabular}

\section{Syndicate 3}

\begin{tabular}{|c|c|c|c|c|c|c|c|c|c|c|c|}
\hline Title & Author & Nat. & 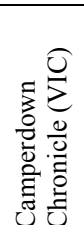 & 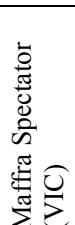 & 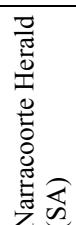 & 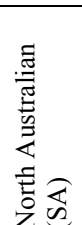 & 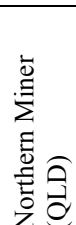 & 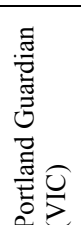 & 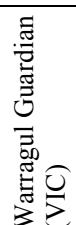 & 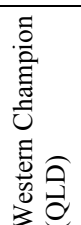 & 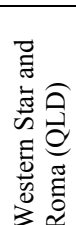 \\
\hline "Griffith Gaunt; Or, Jealousy" (1885) & Charles Reade & Brit & & & & $\mathrm{X}$ & $\mathrm{X}$ & & & & \\
\hline "A Wire Frame" (1886) & Unknown & Unk & & & & $\mathrm{X}$ & & & $\mathrm{X}$ & & \\
\hline "The Elephant Tamer" (1886) & Unknown & Unk & & & & $\mathrm{X}$ & & $\mathrm{X}$ & $\mathrm{X}$ & & \\
\hline $\begin{array}{l}\text { "Arthur Landon, the Australian Bushman" } \\
\text { (1887) }\end{array}$ & Angus McLean & Aust & & $\mathrm{X}$ & & $\mathrm{X}$ & & & $\mathrm{X}$ & $\mathrm{X}$ & \\
\hline "Out of a Labyrinth" (1887) & $\begin{array}{l}\text { Emma Murdoch Van } \\
\text { Deventer }\end{array}$ & $\mathrm{Am}$ & & & $\mathrm{X}$ & $\mathrm{X}$ & & & $\mathrm{X}$ & $\mathrm{X}$ & \\
\hline "The Black Pearl" (1887) & Victorien Sardou & $\mathrm{Fr}$ & & & & $\mathrm{X}$ & & & $\mathrm{X}$ & $\mathrm{X}$ & \\
\hline "The Maiden of Windorf" (1887) & Sylvanus Cobb & $\mathrm{Am}$ & & & & $\mathrm{X}$ & & & $\mathrm{X}$ & & \\
\hline "The Man From Australia" (1887) & Grosvenor Bunster & Aust & & $\mathrm{X}$ & & $\mathrm{X}$ & & & $\mathrm{X}$ & $\mathrm{X}$ & \\
\hline $\begin{array}{l}\text { "A Gilded Bait; Or, Kin But Less Than } \\
\text { Kind" (1888) }\end{array}$ & \# An Old Contributor & Unk & & $\mathrm{X}$ & & $\mathrm{X}$ & & & $\mathrm{X}$ & & $\mathrm{X}$ \\
\hline $\begin{array}{l}\text { "A Late Repentance; Or, The Little White } \\
\text { Hand" (1888) }\end{array}$ & Mary A. Denison & $\mathrm{Am}$ & & & $\mathrm{X}$ & $\mathrm{X}$ & & & $\mathrm{X}$ & & $\mathrm{X}$ \\
\hline "An Evil Reputation" (1888) & Dora Russell & Brit & & & $\mathrm{X}$ & $\mathrm{X}$ & & & $\mathrm{X}$ & & $\mathrm{X}$ \\
\hline "Friends; Or, A Double Trial" (1888) & J. L. N. & Unk & & & & & & & $\mathrm{X}$ & & $\mathrm{X}$ \\
\hline "Ionie, The Pride of the Mill" (1889) & Laura Jean Libbey & $\mathrm{Am}$ & & $\mathrm{X}$ & & $\mathrm{X}$ & & & & & \\
\hline "Mignon; Or, Playing with Fire" (1889) & A. D. N. & Unk & & & & $\mathrm{X}$ & & & $\mathrm{X}$ & & $\mathrm{X}$ \\
\hline "The Squatter's Daughter" (1890) & Harold M. Mackenzie & Aust & $\mathrm{X}$ & $\mathrm{X}$ & & $X$ & & & & & \\
\hline
\end{tabular}


Title

"The Golden Hope" (1886/7)

"Between the Two" (1887)

"Like and Unlike" (1887)

"Black Blood" (1888)

"Dead Man's Rock" (1888)

"The Outlaws of Tunstall Forest" (1888)

"The Pillar House" (1890)

"A Hardy Norseman" (1889/90)

"Commodore Junk" (1888/9)

"My Plucky Boy Tom; Or, Searching for

Curiosities in India for My Show"

(1888/9)

"The Frozen Pirate" (1888/9)

"An Ocean Tragedy" (1890)

"A Smuggler's Secret" (1890)

"By Pike and Dyke" (1890)

"The Bolton Square Tragedy" (1890)

"The Bondman" (1890)

"The Curse of Carne's Hold" (1890)

"The Only Girl At Overlook" (1890)

"The Sign of the Four" (1890)

"Leonard Lindsay; Or, The Story of a Buccaneer" (1890/1)

"Paoli, the Warrior Bishop; Or, The Fall

of the Christians" (1890/1)

"The Missing Merchantman" (1890/1)

"A Final Reckoning" (1891)

"Charlie Ransom" (1891)

"McDermot, the Foundling" (1891)

"Strange Friends" (1891)

"Written in Red; Or, The Conspiracy in

the North Case. A Story of Boston

"His Angel" (1891/2)

"The House of Rimmon" (1891/2)

"The Master of Silence" (1891/2)

"The Right Sort" (1892)

"Brought to Book" (1893)

"The Golden Caves" (1893)
Author

W. Clark Russell

\begin{tabular}{lll|l} 
Unknown & Unk & $\mathrm{X}$ & \\
\hline
\end{tabular}

\begin{tabular}{lllllll|l|l|l|l|l} 
George Manville Fenn & Brit & X & & & X & & & & & X & X \\
\hline
\end{tabular}

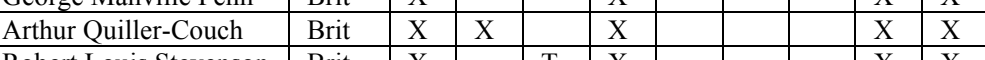

\begin{tabular}{l|l|l|l|l|l} 
Robert Louis Stevenson & Brit & X & & T & X \\
\hline
\end{tabular}

\begin{tabular}{l|l} 
Florence Severne & Unk \\
\hline
\end{tabular}

George Manville Fenn $\quad$ B

\begin{tabular}{ll|l|l|l|l|l|l|l|l|l|l|} 
\\
$\mathrm{X}$ & & & & & $\mathrm{X}$ & $\mathrm{X}$ & & & $\mathrm{X}$ & $\mathrm{X}$ \\
\hline $\mathrm{X}$ & $\mathrm{X}$ & & & $\mathrm{X}$ & $\mathrm{X}$ & $\mathrm{X}$ & & & $\mathrm{X}$ & $\mathrm{X}$ \\
\hline
\end{tabular}

\begin{tabular}{l|l}
\hline P. T. Barnum & $\mathrm{Am}$
\end{tabular}

W. Clark Russell

W. Clark Russell

\begin{tabular}{l|l} 
W. Clark Russell & B \\
\hline Fit & Brit \\
\hline
\end{tabular}

$\mathrm{X} \quad \mathrm{X}$

\begin{tabular}{|l|l|l|l|l|} 
& & & $\mathrm{X}$ \\
\hline $\mathrm{X}$ & $\mathrm{X}$ & & $\mathrm{X}$ \\
\hline
\end{tabular}

\begin{tabular}{ll} 
G. A. Henty & Brit \\
\hline
\end{tabular}

\begin{tabular}{ll|l} 
Brit & & $\mathrm{X}$ \\
\hline Brit & $\mathrm{X}$ & $\mathrm{X}$ \\
\hline
\end{tabular}

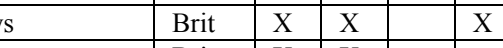

Hall Caine

G. A. Henty

Franklin Fyles

Arthur Conan Doyle

Brit $\quad \mathrm{X} / \mathrm{X}$

Angus B. Reach

W. C. Kitchin

\begin{tabular}{l|l|l|}
\hline Am & $X$ & $X$ \\
\hline
\end{tabular}

\begin{tabular}{|l|l|l|l}
\hline Brit & & $\mathrm{X}$ \\
\hline Brit & &
\end{tabular}

Brit

\begin{tabular}{l|l|l|}
\hline Brit & $\mathrm{X}$ & $\mathrm{X}$
\end{tabular}

\begin{tabular}{l|l|} 
& $\mathrm{X}$ \\
$\mathrm{Am}$ & $\mathrm{X}$
\end{tabular}

Harry Collingwood

G. A. Henty

William Atkinson

Ampere

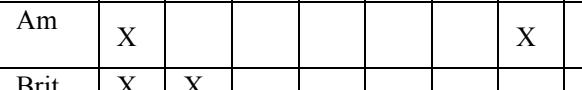

\begin{tabular}{|c|c|c|c|c|} 
Brit & $\mathrm{X}$ & $\mathrm{X}$ & & \\
\hline Brit & $\mathrm{X}$ & $\mathrm{X}$ & & $\mathrm{X}$ \\
\hline
\end{tabular}

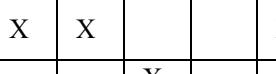

m Atkinson

Charles How
Montague

Henry Herman

\begin{tabular}{ll|l|l|} 
& & $\mathrm{X}$ \\
\hline Unk & $\mathrm{X}$ & $\mathrm{X}$ \\
\hline & $\mathrm{X}$ & $\mathrm{X}$
\end{tabular}

Jeanie Gwynne Bettany

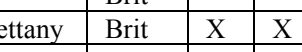

\begin{tabular}{l|l|l|l|}
\hline Irving Bacheller & Am & $X$ & $X$ \\
\hline Mrs Edwar Kennt & Bit & X & \\
\hline
\end{tabular}

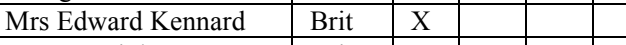

T. W. Speight

Brit

$\mathrm{Am}$

\begin{tabular}{|l|l|l|l|l|}
\hline $\mathrm{X}$ & $\mathrm{X}$ & & $\mathrm{X}$ & \\
\hline $\mathrm{X}$ & & & & \\
\hline
\end{tabular}

\begin{tabular}{|l|l|l|l|l|l|l|}
$\mathrm{X}$ & $\mathrm{X}$ & & & & $\mathrm{X}$ & \\
\hline $\mathrm{X}$ & $\mathrm{X}$ & & $\mathrm{X}$ & & & $\mathrm{X}$ \\
\hline
\end{tabular}

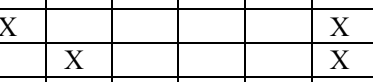

\begin{tabular}{|l|l|l|l|l|l|l|l|l|l|l|}
\hline $\mathrm{X}$ & & & & $\mathrm{X}$ & & & & & & \\
\hline $\mathrm{X}$ & & $\mathrm{X}$ & $\mathrm{X}$ & & & & & & $\mathrm{X}$ & \\
\hline
\end{tabular} \begin{tabular}{|l|l|l|l|l|}
$\mathrm{X}$ & & $\mathrm{X}$ & $\mathrm{X}$ & \\
$\mathrm{X}$ & $\mathrm{T}$ & & & $\mathrm{X}$ \\
\hline
\end{tabular}

\begin{tabular}{|c|c|c|c|c|c|}
\hline & & & & & \\
\hline $\mathrm{X}$ & & & $\mathrm{T}$ & $X$ & $\mathrm{X}$ \\
\hline $\mathrm{X}$ & $\mathrm{X}$ & & & & $\mathrm{X}$ \\
\hline X & $\mathrm{X}$ & & & & $\mathrm{X}$ \\
\hline$X$ & & & $\mathrm{~T}$ & & $\mathrm{X}$ \\
\hline$X$ & $X$ & & $\mathrm{~T}$ & & $\mathrm{X}$ \\
\hline$X$ & & $X$ & $\mathrm{~T}$ & & $\mathrm{X}$ \\
\hline & & $X$ & $\mathrm{~T}$ & $\mathrm{X}$ & $X$ \\
\hline
\end{tabular}

\begin{tabular}{|l|l|l|l|l|l|}
\hline $\mathrm{X}$ & & & & \\
\hline & & & & \\
\hline
\end{tabular} $\mathrm{X}$

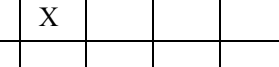

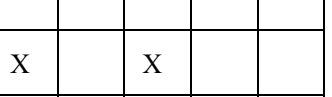

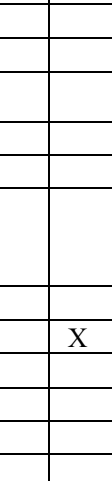

\begin{tabular}{|l|l|l|l}
\hline$X$ & $X$ & $X$ \\
\hline
\end{tabular}

$\mathrm{X}$

\begin{tabular}{|l|l|l|l|l|l|l|l|l|l|l|}
\hline $\mathrm{X}$ & & & $\mathrm{T}$ & $\mathrm{X}$ & & $\mathrm{X}$ & & $\mathrm{X}$ & & \\
\hline $\mathrm{X}$ & & & $\mathrm{T}$ & $\mathrm{X}$ & & $\mathrm{X}$ & $\mathrm{X}$ & $\mathrm{X}$ & & \\
\hline $\mathrm{X}$ & & & $\mathrm{X}$ & & $\mathrm{X}$ & & $\mathrm{X}$ & & \\
\hline
\end{tabular}

\begin{tabular}{|l|l|l|l|l|l|l|l|l|l|l|}
\hline $\mathrm{X}$ & & & $\mathrm{T}$ & $\mathrm{X}$ & & $\mathrm{X}$ & $\mathrm{X}$ & $\mathrm{X}$ & & \\
\hline $\mathrm{X}$ & & & & $\mathrm{X}$ & & $\mathrm{X}$ & & $\mathrm{X}$ & & \\
\hline $\mathrm{X}$ & & & $\mathrm{X}$ & $\mathrm{X}$ & & $\mathrm{X}$ & & & & \\
\hline $\mathrm{X}$ & & & $\mathrm{T}$ & $\mathrm{X}$ & & $\mathrm{X}$ & & $\mathrm{X}$ & & \\
\hline
\end{tabular}

\begin{tabular}{|c|l|l|l|l|l|l|l|l|l|l|}
$\mathrm{X}$ & & & $\mathrm{T}$ & $\mathrm{X}$ & & $\mathrm{X}$ & & $\mathrm{X}$ & & \\
$\mathrm{X}$ & & & & & & $\mathrm{X}$ & & & & \\
\hline
\end{tabular} (

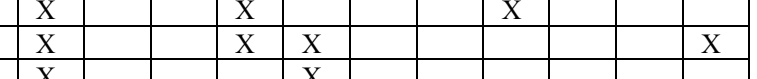
\begin{tabular}{l|l}
$\mathrm{X}$ & \\
$\mathrm{X}$ & $\mathrm{T}$ \\
\hline $\mathrm{X}$ & \\
\hline
\end{tabular}

\begin{tabular}{l|l|l|l|}
$\mathrm{X}$ & $\mathrm{X}$ & & $\mathrm{X}$ \\
$\mathrm{X}$ & $\mathrm{X}$ & & $\mathrm{X}$ \\
\hline
\end{tabular} \begin{tabular}{l|l}
$\mathrm{X}$ & $\mathrm{X}$ \\
\hline
\end{tabular}

$+$ \begin{tabular}{l|l}
$\mathrm{X}$ & \\
\hline &
\end{tabular} 


\begin{tabular}{|c|c|c|c|c|c|c|c|c|c|c|c|c|c|}
\hline Title & Author & Nat. & 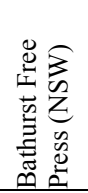 & 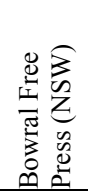 & 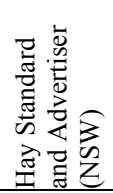 & 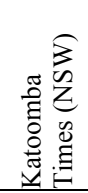 & 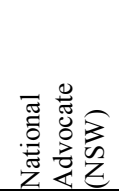 & 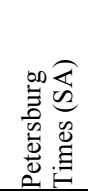 & 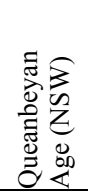 & 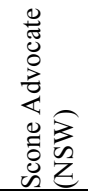 & 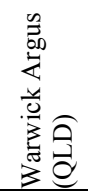 & 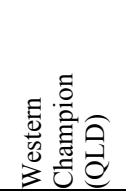 & 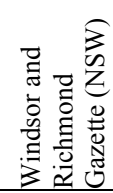 \\
\hline "Goths, Huns, and Vandals" (1887) & Harold W. H. Stephen & Aust & & $\mathrm{X}$ & & & & & $\mathrm{X}$ & $\mathrm{X}$ & & & \\
\hline "The Golden Yankee" (1887) & Harold W. H. Stephen & Aust & $\mathrm{X}$ & $\mathrm{X}$ & & & & & $\mathrm{X}$ & & & & \\
\hline "For Lack of Gold" (1888) & \# M. B. Simmons & Unk & & $\mathrm{X}$ & & & & $\mathrm{X}$ & $\mathrm{X}$ & $\mathrm{X}$ & & & \\
\hline "Margret, the Story of a Crime" (1888) & Hans Blum & Ger & & $\mathrm{X}$ & & & & $\mathrm{X}$ & $\mathrm{X}$ & $\mathrm{T}$ & & $\mathrm{X}$ & \\
\hline "Bella-Demonia" (1889) & Selina Dolaro & Brit & $\mathrm{X}$ & & & $\mathrm{X}$ & $\mathrm{X}$ & $\mathrm{X}$ & & & & & $\mathrm{X}$ \\
\hline "Dunraven Ranch" (1889) & Charles King & $\mathrm{Am}$ & $\mathrm{X}$ & $\mathrm{X}$ & & & & $\mathrm{X}$ & & & & $\mathrm{X}$ & $\mathrm{X}$ \\
\hline "Hale-Weston" (1889) & Molly Elliot Seawell & $\mathrm{Am}$ & $\mathrm{X}$ & & & & & $\mathrm{X}$ & & $\mathrm{X}$ & & $\mathrm{X}$ & $\mathrm{X}$ \\
\hline "The New Editor" (1889/90) & Harold W. H. Stephen & Aust & $\mathrm{X}$ & & & $\mathrm{X}$ & & $\mathrm{X}$ & & & & & $\mathrm{X}$ \\
\hline "That Girl From Texas" (1889/90) & Jeannette H. Walworth & $\mathrm{Am}$ & $\mathrm{X}$ & & & $\mathrm{X}$ & & & & $\mathrm{X}$ & $\mathrm{X}$ & & $\mathrm{X}$ \\
\hline "Frances Kane's Fortune" (1890) & L. T. Meade & Brit & $\mathrm{X}$ & & & & & $\mathrm{X}$ & & $\mathrm{X}$ & & & $\mathrm{X}$ \\
\hline "Miss Ludington’s Sister" (1890) & Edward Bellamy & $\mathrm{Am}$ & $\mathrm{X}$ & & & $\mathrm{X}$ & & $\mathrm{X}$ & & $\mathrm{X}$ & & & $\mathrm{X}$ \\
\hline "A Cast for Fortune" (1891) & Christian Reid & $\mathrm{Am}$ & & & & & & $\mathrm{X}$ & & $\mathrm{X}$ & & & $\mathrm{X}$ \\
\hline "A Rose of a Hundred Leaves" (1891) & Amelia E. Barr & Brit & & & & & & $\mathrm{X}$ & & $\mathrm{X}$ & & & $\mathrm{X}$ \\
\hline "A Sappho of Green Springs" (1891) & Bret Harte & $\mathrm{Am}$ & & & & & & $\mathrm{X}$ & & $\mathrm{X}$ & & & $\mathrm{X}$ \\
\hline "A Wave of Life" (1891) & Clyde Fitch & $\mathrm{Am}$ & & & & & $\mathrm{X}$ & $\mathrm{X}$ & & & & & $\mathrm{X}$ \\
\hline "By the World Forgot" (1891) & E. J. Clayden & Unk & $\mathrm{X}$ & & & & $\mathrm{X}$ & $\mathrm{X}$ & & $\mathrm{X}$ & & & $\mathrm{X}$ \\
\hline “A Fair Blockade Breaker” (1892) & T. C. De Leon & $\mathrm{Am}$ & & & $\mathrm{X}$ & & & $\mathrm{X}$ & & $\mathrm{X}$ & & & \\
\hline "Lady Patty" (1892) & $\begin{array}{l}\text { Margaret Wolfe } \\
\text { Hungerford }\end{array}$ & Brit & & & & & & $\mathrm{X}$ & & $\mathrm{X}$ & & & \\
\hline "The Italian Peasant" (1892) & Unknown & Unk & & & & & & $\mathrm{X}$ & & & & & $\mathrm{X}$ \\
\hline "The House on the Marsh" (1893) & Florence Warden & Brit & $\mathrm{X}$ & $\mathrm{X}$ & & & & & & & & & \\
\hline
\end{tabular}

\section{Syndicate 6}

\begin{tabular}{|c|c|c|c|c|c|c|c|c|c|c|c|c|c|c|c|c|c|c|c|c|c|c|c|c|c|c|c|c|c|c|c|c|c|c|c|c|c|c|c|c|c|}
\hline Title & Author & Nat. & & $\begin{array}{r}2 \\
0 \\
0 \\
0 \\
0 \\
0 \\
0 \\
0 \\
0 \\
0 \\
0 \\
0 \\
0 \\
0 \\
0 \\
0 \\
0 \\
0 \\
0\end{array}$ & $\begin{array}{l}0 \\
0 \\
0 \\
0 \\
0 \\
0 \\
0 \\
0 \\
0 \\
0 \\
0\end{array}$ & $\begin{array}{l}0 \\
0 \\
0 \\
0 \\
0 \\
0 \\
0 \\
0 \\
0 \\
0 \\
0 \\
0 \\
0 \\
0 \\
0 \\
0\end{array}$ & $\begin{array}{l}0 \\
3 \\
0 \\
0 \\
0 \\
0 \\
0 \\
0 \\
0 \\
0 \\
0 \\
0 \\
0 \\
0\end{array}$ & $\begin{array}{l} \\
0 \\
0 \\
0 \\
0 \\
0 \\
0 \\
0 \\
0 \\
0 \\
0 \\
0\end{array}$ & 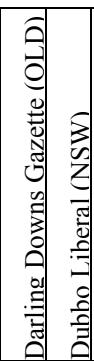 & $\begin{array}{l}\mid \\
3 \\
0 \\
z \\
0 \\
0 \\
0 \\
0 \\
0 \\
0 \\
0 \\
0 \\
0 \\
0\end{array}$ & 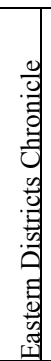 & $\begin{array}{l}0 \\
0 \\
0 \\
0 \\
0 \\
0 \\
0 \\
0 \\
0 \\
0 \\
0 \\
0\end{array}$ & 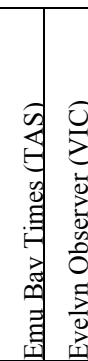 & 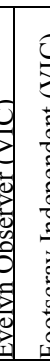 & 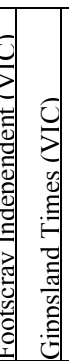 & 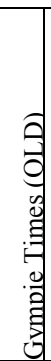 & 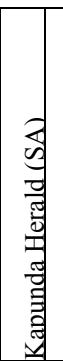 & 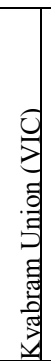 & 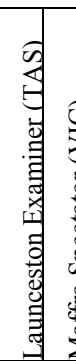 & 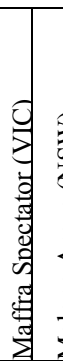 & 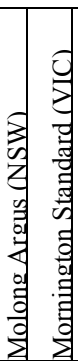 & 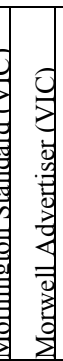 & 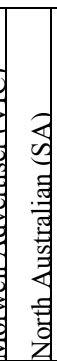 & 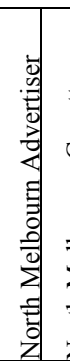 & 基 & 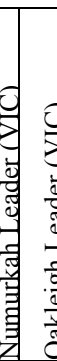 & 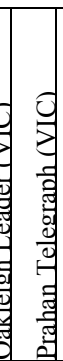 & 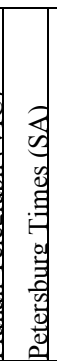 & 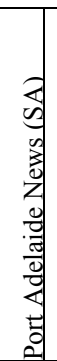 & 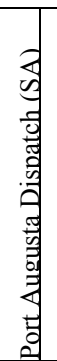 & $\begin{array}{l}0 \\
0 \\
0 \\
0 \\
0 \\
0 \\
0 \\
0 \\
0 \\
0 \\
0 \\
0 \\
0\end{array}$ & 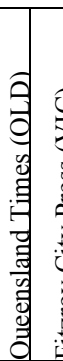 & 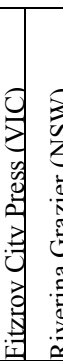 & 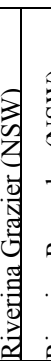 & 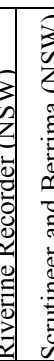 & 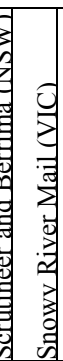 & \begin{tabular}{|}
0 \\
0 \\
0 \\
0 \\
0 \\
0 \\
0 \\
0 \\
0 \\
0
\end{tabular} & 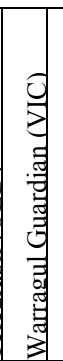 & 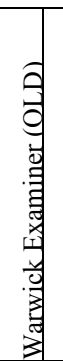 & 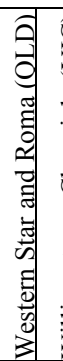 & 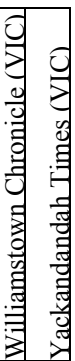 \\
\hline $\begin{array}{l}\text { "The Masterpiece of Crime" } \\
(1891 / 2)\end{array}$ & Jean Richepin & Fre & & & & & $\mathrm{x}$ & $\mathrm{x}$ & & & & & & & & & & & & & $x$ & $x$ & & 4 & $\frac{4}{x}$ & & $\mathrm{x}$ & & & & & & & & & & & & & & $\mathrm{x}$ \\
\hline $\begin{array}{l}\text { "An Eventful Night; Or, the } \\
\text { Captain's Peril" (1892) }\end{array}$ & F. G. Kerr & Unk & $\mathrm{x}$ & & & & & & & & & & & & & & & & & & & & & & & & & & & & & & & & & $\mathrm{X}$ & & & & & \\
\hline $\begin{array}{l}\text { "Fairy Queen; Or, Love Will } \\
\text { Find the Way" (1892/3) }\end{array}$ & Bonnie Dene & Unk & $\mathrm{X}$ & & & & & & & & & & & & & & & & & & & & & & & & & & & & & & & & & $\mathrm{X}$ & & & & & \\
\hline "Maoriland Ho!" (1892/3) & Atha Westbury & Aust & & & $\mathrm{x}$ & $\mathrm{X}$ & & & & & $\mathrm{X}$ & & $\mathrm{X}$ & & & & & & & & & & $\mathrm{X}$ & & & & & & $\mathrm{X}$ & & & & & $\bar{x}$ & & & & & & & \\
\hline "Seed-Time and Harvest" (1892) & M. A. & Aust & & & & & $\mathrm{X}$ & $\mathrm{X}$ & & & & & & & & & & $\mathrm{X}$ & & & $\mathrm{X}$ & & & $\mathrm{T}$ & & & $\mathrm{X}$ & & & & & & & & & & & $\mathrm{X}$ & & & \\
\hline $\begin{array}{l}\text { "The Avenging Skeleton" } \\
(1892 / 3)\end{array}$ & $\begin{array}{l}\text { E. Branfill Harrison } \\
\text { and Gustav } \\
\text { Toudouze }\end{array}$ & Fre & & & & & $\mathrm{X}$ & $\mathrm{x}$ & & & & & & & & & & & & & $\mathrm{x}$ & & & & & & $\mathrm{x}$ & & & & & & & & & & & & & & \\
\hline "The Fatal Treasure" (1892/3) & James J. Wright & Aust & $\mathrm{X}$ & & $\mathrm{X}$ & $\mathrm{X}$ & & & & & $\mathrm{X}$ & & $\mathrm{X}$ & & & $\mathrm{X}$ & $\mathrm{X}$ & & & $\mathrm{X}$ & & & & & & & & & $\mathrm{X}$ & & $\mathrm{X}$ & & $\mathrm{X}$ & & & & & & $\mathrm{X}$ & & \\
\hline
\end{tabular}









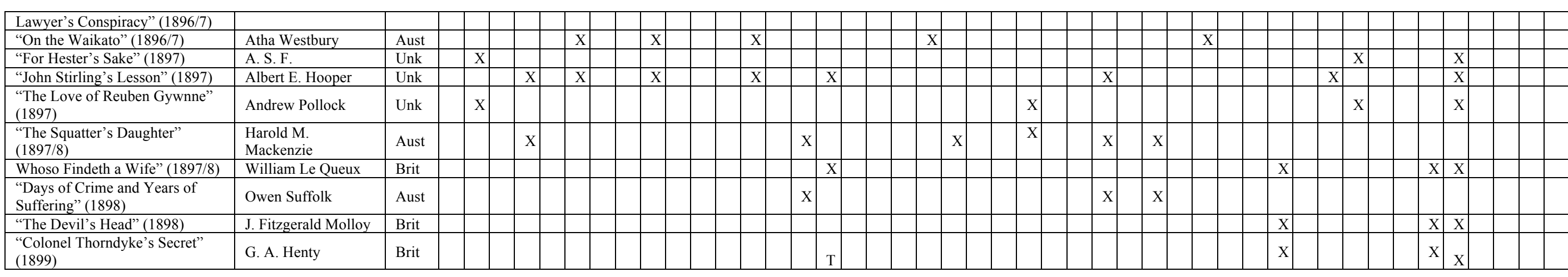

\section{Syndicate 7}

\begin{tabular}{|c|c|c|c|c|c|c|c|c|c|c|c|c|c|c|c|}
\hline Title & Author & Nat. & 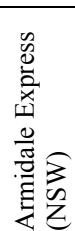 & 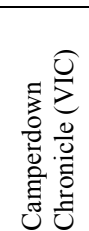 & 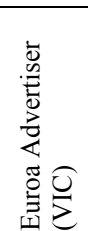 & 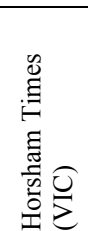 & 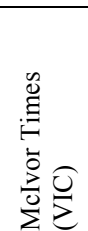 & 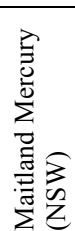 & 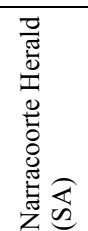 & 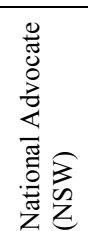 & 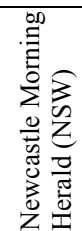 & 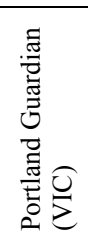 & 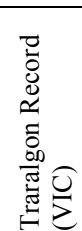 & 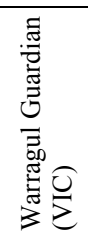 & 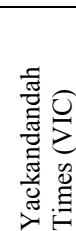 \\
\hline "Love Better Than Sacrifice" (1892) & Oscar Park & Unk & $\mathrm{X}$ & $\mathrm{X}$ & & $\mathrm{X}$ & $\mathrm{X}$ & & & $\mathrm{X}$ & & $\mathrm{X}$ & $\mathrm{X}$ & & \\
\hline $\begin{array}{l}\text { "Found Guilty; Or, The Hidden Crime" } \\
(1892 / 3)\end{array}$ & Leon Lewis & $\mathrm{Am}$ & & $\mathrm{X}$ & $\mathrm{X}$ & $\mathrm{X}$ & $\mathrm{X}$ & & $\mathrm{X}$ & & & $\mathrm{X}$ & $\mathrm{X}$ & & \\
\hline "Her Colonial Cousin" (1893) & Unknown & Unk & & $\mathrm{X}$ & $\mathrm{X}$ & & $\mathrm{X}$ & & $\mathrm{X}$ & & & & & & \\
\hline "The Mystery of the Tower" (1893) & Helen Corwin Pierce & $\mathrm{Am}$ & & $\mathrm{X}$ & $\mathrm{X}$ & $\mathrm{X}$ & $\mathrm{X}$ & & $\mathrm{X}$ & & & $\mathrm{X}$ & $\mathrm{X}$ & $\mathrm{X}$ & \\
\hline "The Story of Andrew Fairfax" (1893) & Joseph Hocking & Brit & & $\mathrm{X}$ & $\mathrm{X}$ & $\mathrm{X}$ & $\mathrm{X}$ & & $\mathrm{X}$ & & $\mathrm{X}$ & $\mathrm{X}$ & & & \\
\hline "Ishmael Pengelly" (1893/4) & Joseph Hocking & Brit & & & $\mathrm{X}$ & $\mathrm{X}$ & $\mathrm{X}$ & & $\mathrm{X}$ & & $\mathrm{X}$ & $\mathrm{X}$ & & & \\
\hline "A Dead Man’s Step" (1894) & Lawrence L. Lynch & $\mathrm{Am}$ & & & $\mathrm{X}$ & $\mathrm{X}$ & $\mathrm{X}$ & & $\mathrm{X}$ & & & $\mathrm{X}$ & & & \\
\hline "In Strange Company" (1894/5) & Guy Boothby & Aust & & & $\mathrm{X}$ & $\mathrm{X}$ & $\mathrm{X}$ & & $\mathrm{X}$ & & $\mathrm{X}$ & $\mathrm{X}$ & & & \\
\hline "The King of Hearts" (1894/5) & Outram Tristram & Brit & & & $\mathrm{X}$ & $\mathrm{X}$ & & & $\mathrm{X}$ & & $\mathrm{X}$ & $\mathrm{X}$ & & $\mathrm{X}$ & \\
\hline "A Mistaken Man" (1895) & Nick Carter & $\mathrm{Am}$ & & & $\mathrm{X}$ & & & & $\mathrm{X}$ & & & $\mathrm{X}$ & & & \\
\hline "All Men Are Liars" (1895) & Joseph Hocking & Brit & & $\mathrm{X}$ & $\mathrm{X}$ & $\mathrm{X}$ & & & $\mathrm{X}$ & & & $\mathrm{X}$ & & & \\
\hline $\begin{array}{l}\text { "Her Three Lovers; Or, Delauney's Debt" } \\
\text { (1895) }\end{array}$ & Unknown & Unk & & & $\mathrm{X}$ & & & & $\mathrm{X}$ & & & $\mathrm{X}$ & & & \\
\hline "Kerrigan’s Quality" (1895) & Jane Barlow & Brit & & $\mathrm{X}$ & $\mathrm{X}$ & & & & $\mathrm{X}$ & & & $\mathrm{X}$ & & & \\
\hline "My Gifted Young Friend" (1895) & Nick Carter & $\mathrm{Am}$ & & & $\mathrm{X}$ & & & & $\mathrm{X}$ & & & $\mathrm{X}$ & & & \\
\hline "The Great Hesper" (1895) & Frank Barrett & Brit & & & $\mathrm{X}$ & & & & $\mathrm{X}$ & & & $\mathrm{X}$ & & & \\
\hline "The Menacing Eye" (1895) & Nick Carter & $\mathrm{Am}$ & & & $\mathrm{X}$ & & & & $\mathrm{X}$ & & & $\mathrm{X}$ & & & \\
\hline "The Sacred Beetle" (1895) & Lamont Graeme & Unk & & & $\mathrm{X}$ & & & & $\mathrm{X}$ & & & $\mathrm{X}$ & & & \\
\hline "A Lady in Black" (1895/6) & Florence Warden & Brit & & & $\mathrm{X}$ & $\mathrm{X}$ & $\mathrm{X}$ & & $\mathrm{X}$ & & & $\mathrm{X}$ & & & $\mathrm{X}$ \\
\hline "The Crime of the "Liza Jane"" (1896) & Fergus Hume & Brit & & $\mathrm{X}$ & $\mathrm{X}$ & & & & & & & $\mathrm{X}$ & & & \\
\hline "The Hostess of the Griffin" (1896) & Florence Warden & Brit & & & $\mathrm{X}$ & & & & & & & $\mathrm{X}$ & & & \\
\hline $\begin{array}{l}\text { "The Senfrey Mystery; Or, A Brother's } \\
\text { Vengeance" (1896) }\end{array}$ & Henry Herman & Brit & & & $\mathrm{X}$ & & & & & & & $\mathrm{X}$ & & & \\
\hline "Travelling with Half a Million" (1896) & Unknown & Unk & & & $\mathrm{X}$ & & & & & & & $\mathrm{X}$ & & & \\
\hline "Fields of Fair Renown" (1896/7) & Joseph Hocking & Brit & & & $\mathrm{X}$ & $\mathrm{X}$ & & & $\mathrm{X}$ & & & & & & \\
\hline "His Sweetheart Eliza" (1897) & Margaret Moule & Brit & & & & $\mathrm{X}$ & $\mathrm{X}$ & & $\mathrm{X}$ & & & & & & \\
\hline "In the Dead of Night" (1897) & Ada Cambridge & Aust & & & $\mathrm{X}$ & $\mathrm{X}$ & $\mathrm{X}$ & & $\mathrm{X}$ & & & & & & \\
\hline "The Mystery of a Name" (1897) & Unknown & Unk & & & $\mathrm{X}$ & $\mathrm{X}$ & $\mathrm{X}$ & & $\mathrm{X}$ & & & & & & \\
\hline "Two Old Fogies" (1897) & Ada Cambridge & Aust & & $\mathrm{X}$ & $\mathrm{X}$ & & $\mathrm{X}$ & & $\mathrm{X}$ & & & & & & \\
\hline
\end{tabular}




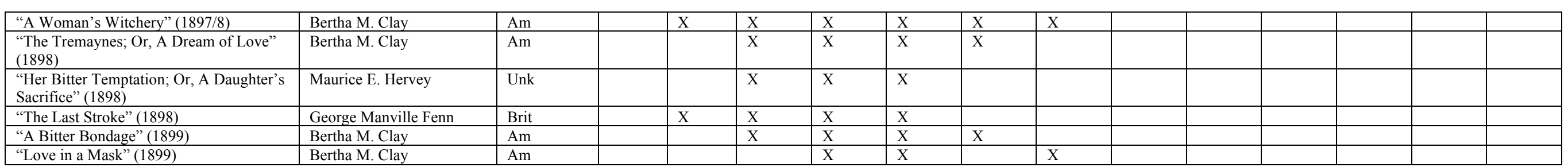

\section{Syndicate 8}

\begin{tabular}{|c|c|c|c|c|c|c|c|c|c|c|c|c|c|c|c|c|c|c|c|c|c|c|c|c|c|}
\hline Title & Author & Nat. & $\begin{array}{l}0 \\
0 \\
0 \\
0 \\
0 \\
0 \\
0 \\
0\end{array}$ & 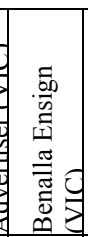 & 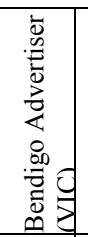 & 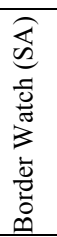 & 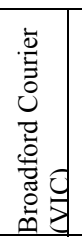 & 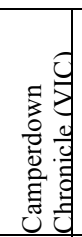 & 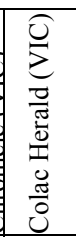 & 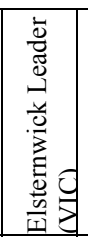 & 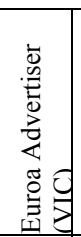 & 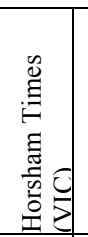 & 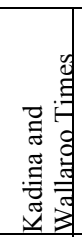 & 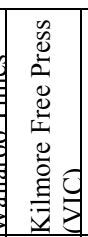 & 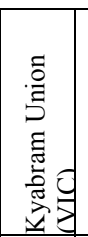 & 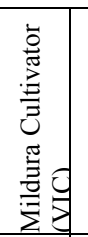 & 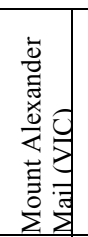 & 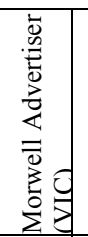 & 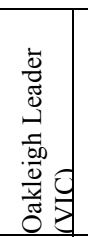 & 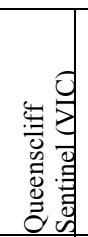 & 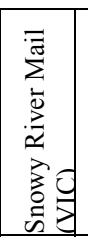 & 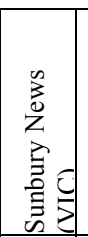 & 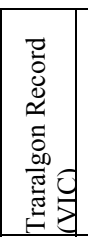 & 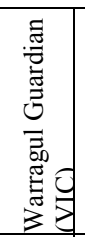 & 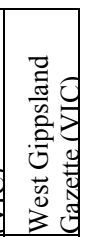 \\
\hline "An Original Temptation" (1892) & Marquise Clara Lanza & $\mathrm{Am}$ & $\bar{X}$ & & & $\mathrm{X}$ & & & & & & & & $\mathrm{X}$ & & & & & & & $\mathrm{X}$ & & & & \\
\hline "Greymere" (1892) & Unknown & Unk & & & & $\mathrm{X}$ & & & & & & & & $\mathrm{X}$ & & $\mathrm{X}$ & & & & & & & & & \\
\hline "My Poor Little Story" (1892) & Unknown & Unk & & & & & & & & & & $\mathrm{X}$ & & & & & & & & & $\mathrm{X}$ & & $\mathrm{X}$ & & \\
\hline "On Suspicion" (1892) & Unknown & Unk & & & & $\mathrm{X}$ & & & & & & & & & & & & & & & $\mathrm{X}$ & & & & \\
\hline "The Doctor's Secret" (1892) & M. H. & Unk & & & & $\mathrm{X}$ & & & & & & & & & & & & & & & $\mathrm{X}$ & & & & \\
\hline "A Coming Possibility" (1893) & Greg Edwards & $\mathrm{Am}$ & & & & & $\mathrm{X}$ & $\mathrm{X}$ & & & & & & & & & & & & & & & & $\mathrm{X}$ & \\
\hline "By Accident" (1893) & H. F. Abell & Unk & & & & & & $\mathrm{X}$ & & & & & & & & & & & & & & & $\mathrm{X}$ & $\mathrm{X}$ & \\
\hline $\begin{array}{l}\text { "Faithful Shirley; Or, A Royal Queen of Hearts" } \\
\text { (1893) }\end{array}$ & Mrs G. Sheldon & $\mathrm{Am}$ & $\mathrm{X}$ & & & $\mathrm{X}$ & & & & & & & & & & & & & & & & $\mathrm{X}$ & $\mathrm{X}$ & & \\
\hline $\begin{array}{l}\text { "Her Life's Desire; Or, A True Heart’s } \\
\text { Sacrifice" (1893) }\end{array}$ & Mrs E. Burke Collins & $\mathrm{Am}$ & $\mathrm{X}$ & & & $\mathrm{X}$ & & & & & & $\mathrm{X}$ & & & & & & & & & & & & & \\
\hline $\begin{array}{l}\text { "Indro; The Man of Iron! Or, The Swift Shadow } \\
\text { of the Rails" (1893) }\end{array}$ & Donald J. McKenzie & $\mathrm{Am}$ & $\mathrm{X}$ & & & $\mathrm{X}$ & & & & & & & & & & & & & & & & & $\mathrm{X}$ & & \\
\hline "John Upcraft's Crime" (1893) & Thomas St. E. Hake & Brit & & & & & $\mathrm{X}$ & $\mathrm{X}$ & & & & & & & & & & & & & & & & & \\
\hline "The Hint o' Hairst" (1893) & Menie Muriel Dowie & Brit & & & & & & $\mathrm{X}$ & & & & & & & & & & & & & & & $\mathrm{X}$ & $\mathrm{X}$ & \\
\hline "The Irony of Fate" (1893) & T. E. Southee & Unk & & & & & & $\mathrm{X}$ & & & & & & & $\mathrm{X}$ & & & & & & & & $\mathrm{X}$ & $\mathrm{X}$ & \\
\hline "The Sacred Beetle"(1893) & Lamont Graeme & Unk & & & & & & $\mathrm{X}$ & & & & & & & & & & & & & & & & $\mathrm{X}$ & \\
\hline "The Street of Peace" (1893) & Oscar Fay Adams & $\mathrm{Am}$ & & & & & & $\mathrm{X}$ & & & & & & & & & & & & & & & & $\mathrm{X}$ & \\
\hline "The Squire's Dilemma" (1893/4) & J. MacLaren Cobban & Brit & & & $\mathrm{X}$ & & & $\mathrm{X}$ & & & & & & & & & & & & & & & $\mathrm{X}$ & $\mathrm{X}$ & \\
\hline "A Broken Past" (1894) & Henrietta Stannard & Brit & & & & & & & & $\mathrm{X}$ & & & & & & & $\mathrm{X}$ & & $\mathrm{X}$ & $\mathrm{X}$ & & & & & \\
\hline "Grazia’s Mistake" (1894) & Mrs Georgie Sheldon & $\mathrm{Am}$ & $\mathrm{X}$ & & & & & & & & & & & & & & & & & & & & $\mathrm{X}$ & $\mathrm{X}$ & \\
\hline "Pomona" (1894) & Evelyn Whitaker & Brit & & & & & $\mathrm{X}$ & $\mathrm{X}$ & & & & & & & & & & & & & & & & $\mathrm{X}$ & \\
\hline "The Dead Gallant" (1894) & Outram Tristram & Brit & $\mathrm{X}$ & & & & & & & & & & & & & & & & & & & & $\mathrm{X}$ & $\mathrm{X}$ & \\
\hline "The Masterpiece of Crime" (1894) & Jean Richepin & Fre & & & & & & & & & & & & & & & & $\mathrm{X}$ & & & & & $\mathrm{X}$ & & \\
\hline "Winning a Wife in Australia" (1894) & A. Donnison & Aust & $\mathrm{X}$ & & & & & & & & & & & & & & & & & & & $\mathrm{X}$ & $\mathrm{X}$ & $\mathrm{X}$ & \\
\hline "At Market Value" (1894/5) & Grant Allen & Can & & & & & $\mathrm{X}$ & $\mathrm{X}$ & & $\mathrm{X}$ & & & & & & & $\mathrm{X}$ & & $\mathrm{X}$ & $\mathrm{X}$ & & & & $\mathrm{X}$ & \\
\hline "Elsie" (1894/5) & John Stafford & Unk & & & & & $\mathrm{X}$ & $\mathrm{X}$ & & & & & & & & & & & & & & & & $\mathrm{X}$ & \\
\hline "That Mysterious Sovereign" (1895) & Unknown & Unk & $\mathrm{X}$ & & & $\mathrm{X}$ & & & & & & & & & & & & & & & & & $\mathrm{X}$ & & \\
\hline $\begin{array}{l}\text { "A Fair Spiritualist; Or, A Woman's Victory" } \\
(1895)\end{array}$ & Erica & Unk & & & & & $\mathrm{X}$ & $\mathrm{X}$ & & & & & & & & & & & & & & & & $\mathrm{X}$ & \\
\hline $\begin{array}{l}\text { "Dora Deene; Or, Neither Wife Nor Widow" } \\
\text { (1895) }\end{array}$ & Bertha M. Clay & $\mathrm{Am}$ & $\mathrm{X}$ & & & $\mathrm{X}$ & & & & & & & & & & & & & & & & & $\mathrm{X}$ & & \\
\hline "The Angel of the Four Corners" (1895) & Gilbert Parker & Can & & & & & $\mathrm{X}$ & & & $\mathrm{X}$ & & & & & & & & & $\mathrm{X}$ & & & & & $\mathrm{X}$ & \\
\hline "The Dance at the Four Corners" (1895) & G. B. Burgin & Brit & & & & & $\mathrm{X}$ & $\mathrm{X}$ & & $\mathrm{X}$ & & & & & & & & & $\mathrm{X}$ & & & & & $\mathrm{X}$ & \\
\hline "The Disappearance of Mr. Derwent" (1895) & Thomas Cobb & Brit & $\mathrm{X}$ & & & $\mathrm{X}$ & & & & & & & & & & & & & & & & & $\mathrm{X}$ & & \\
\hline "A Romance of the Law" (1895) & Sidney Lanan Blanchard & Brit & $\mathrm{X}$ & & & $\mathrm{X}$ & & & & & & & & & & & & & & & & & $\mathrm{X}$ & & \\
\hline $\begin{array}{l}\text { "Dora Deene; Or, Neither Wife Nor Widow" } \\
(1895)\end{array}$ & Bertha M. Clay & $\mathrm{Am}$ & $\mathrm{X}$ & & & $\mathrm{X}$ & & & & & & & & & & & & & & & & & $\mathrm{X}$ & & \\
\hline
\end{tabular}




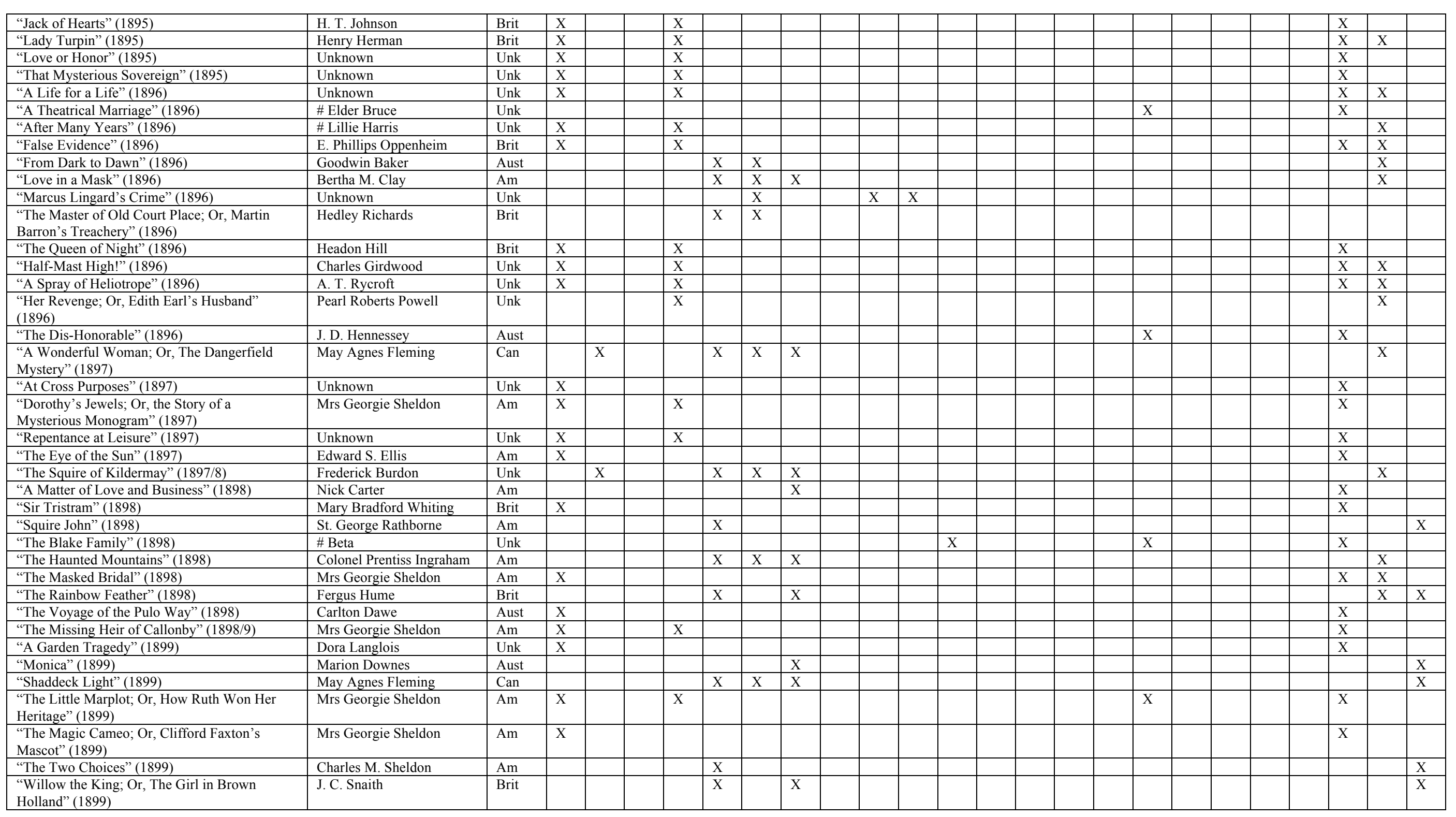




\section{Syndicate 9}

\begin{tabular}{|c|c|c|c|c|c|c|c|c|c|c|c|c|c|c|c|}
\hline Title & Author & Nat. & 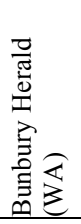 & 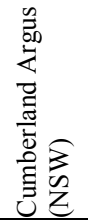 & 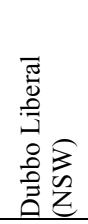 & 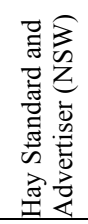 & 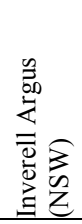 & 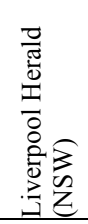 & 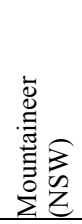 & 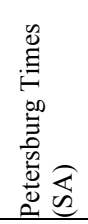 & 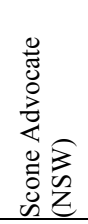 & 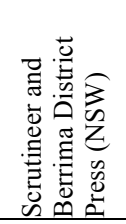 & 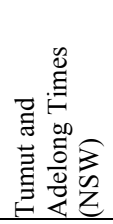 & 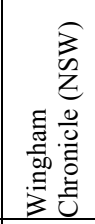 & 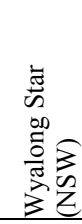 \\
\hline "John Gray" (1893) & James Lane Allen & $\mathrm{Am}$ & & & & $\mathrm{X}$ & & & & & $\mathrm{X}$ & & & & \\
\hline "The Bondswoman" (1893) & Gertrude Atherton & $\mathrm{Am}$ & $\mathrm{X}$ & & & $\mathrm{X}$ & & & & & $\mathrm{X}$ & & & & \\
\hline "The Golden Fleece" (1893) & Julian Hawthorne & $\mathrm{Am}$ & $\mathrm{X}$ & & & $\mathrm{X}$ & & & & & $\mathrm{X}$ & & & & \\
\hline "Mrs Romney" (1893/4) & Rosa Carey & Brit & & $\mathrm{X}$ & & & & & & $\mathrm{X}$ & & $\mathrm{X}$ & & & \\
\hline "A Pacific Encounter" (1894) & Mary E. Stickney & $\mathrm{Am}$ & & $\mathrm{X}$ & & & & & & $\mathrm{X}$ & & $\mathrm{X}$ & & & \\
\hline "Curlew, or in Days Gone By" (1894) & Harold Brees & Aust & & $\mathrm{X}$ & & & & & & $\mathrm{X}$ & & $\mathrm{X}$ & & & $\mathrm{X}$ \\
\hline "Sergeant Croesus" (1894) & Charles King & $\mathrm{Am}$ & & & & $\mathrm{X}$ & & & & & $\mathrm{X}$ & & & & \\
\hline $\begin{array}{l}\text { "The Autobiography of a Professional } \\
\text { Beauty" (1894) }\end{array}$ & Elizabeth Train & $\mathrm{Am}$ & $\mathrm{X}$ & & & $\mathrm{X}$ & & & & & $\mathrm{X}$ & & & & \\
\hline "The Duke and the Commoner" (1894) & Poultney Bigelow & $\mathrm{Am}$ & $\mathrm{X}$ & & & $\mathrm{X}$ & & & & & $\mathrm{X}$ & & & & \\
\hline "The Colonel" (1894) & Harry Willard French & $\mathrm{Am}$ & & $\mathrm{X}$ & & & & & & $\mathrm{X}$ & & $\mathrm{X}$ & & & \\
\hline "Mrs Hallam's Companion" (1895) & Mrs. Mary J. Holmes & $\mathrm{Am}$ & & $\mathrm{X}$ & & $\mathrm{X}$ & & & & $\mathrm{X}$ & & $\mathrm{X}$ & & & \\
\hline "Picotin" (1895) & Alethea Phillips & Unk & & $\mathrm{X}$ & & & & & & $\mathrm{X}$ & & $\mathrm{X}$ & & & \\
\hline "Sweetheart Manette" (1895) & Maurice Thompson & $\mathrm{Am}$ & & $\mathrm{X}$ & & & & & & $\mathrm{X}$ & & $\mathrm{X}$ & & & \\
\hline "An Unsatisfactory Lover" (1895/6) & $\begin{array}{l}\text { Margaret Wolfe } \\
\text { Hungerford }\end{array}$ & Brit & $\mathrm{X}$ & & & $\mathrm{X}$ & & & $\mathrm{X}$ & $\mathrm{X}$ & & $\mathrm{X}$ & & & \\
\hline "Dora's Defiance" (1896) & Lady Lindsay & Brit & & & & $\mathrm{X}$ & & & & $\mathrm{X}$ & & $\mathrm{X}$ & & & \\
\hline "The Troublesome Lady" (1896) & Patience Stapleton & $\mathrm{Am}$ & & & & $\mathrm{X}$ & & & & $\mathrm{X}$ & & & & & \\
\hline "A Bachelor’s Bridal” (1896/7) & Mrs H. Lovett Cameron & Brit & $\mathrm{X}$ & & & $\mathrm{X}$ & & & & $\mathrm{X}$ & & & & & \\
\hline "A Whim and a Chance" (1897) & William T. Nichols & $\mathrm{Am}$ & & & $\mathrm{X}$ & $\mathrm{X}$ & & & & & & & & & \\
\hline "A Question of Courage" (1898) & Francis Lynde & $\mathrm{Am}$ & & & & $\mathrm{X}$ & & $\mathrm{X}$ & & & & $\mathrm{X}$ & & $\mathrm{X}$ & \\
\hline "Flotsam" (1898) & Owen Hall & Brit & & & & $\mathrm{X}$ & & $\mathrm{X}$ & & & $\mathrm{X}$ & & & & \\
\hline "The Bon Marche" (1898) & Unknown & Unk & & & & $\mathrm{X}$ & & $\mathrm{X}$ & & & & & & & \\
\hline "The Chapel of Ease" (1898) & Harriet Riddle Davis & $\mathrm{Am}$ & & & $\mathrm{X}$ & $\mathrm{X}$ & $\mathrm{X}$ & $\mathrm{X}$ & & & & $\mathrm{X}$ & & & \\
\hline "The Daughters of Eve" (1898) & Edwin Doidge & Aust & & & $\mathrm{X}$ & $\mathrm{X}$ & & $\mathrm{X}$ & & & $\mathrm{X}$ & $\mathrm{X}$ & & $\mathrm{X}$ & \\
\hline "Marian Gonisby" (1899) & Edwin Doidge & Aust & & & $\mathrm{X}$ & $\mathrm{X}$ & $\mathrm{X}$ & $\mathrm{X}$ & $\mathrm{X}$ & & $\mathrm{X}$ & $\mathrm{X}$ & $\mathrm{X}$ & $\mathrm{X}$ & \\
\hline "The Martlet Seal" (1899) & Jeanette Walworth & $\mathrm{Am}$ & & & $\mathrm{X}$ & $\mathrm{X}$ & & $\mathrm{X}$ & $\mathrm{X}$ & & & $\mathrm{X}$ & $\mathrm{X}$ & $\mathrm{X}$ & \\
\hline "The Wonder-Witch" (1899) & $\begin{array}{l}\text { Mary Greenway } \\
\text { McClelland }\end{array}$ & $\mathrm{Am}$ & & & $\mathrm{X}$ & & & $\mathrm{X}$ & $\mathrm{X}$ & & $\mathrm{X}$ & $\mathrm{X}$ & $\mathrm{X}$ & $\mathrm{X}$ & \\
\hline "The Yellow Domino" (1899) & M. Morris & Unk & & & $\mathrm{X}$ & $\mathrm{X}$ & & $\mathrm{X}$ & $\mathrm{X}$ & & & & $\mathrm{X}$ & $\mathrm{X}$ & \\
\hline
\end{tabular}




\section{Syndicate 10}

\begin{tabular}{|c|c|c|c|c|c|c|c|c|c|c|c|c|c|c|c|c|c|c|c|c|c|c|c|}
\hline Title & Author & Nat. & 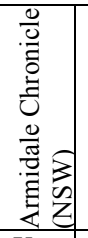 & 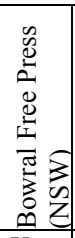 & 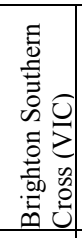 & 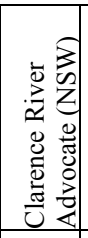 & 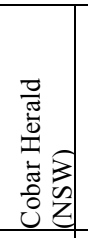 & 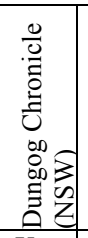 & 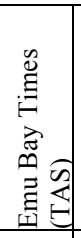 & 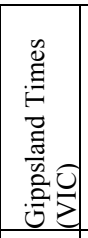 & 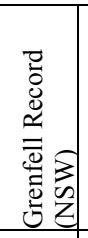 & 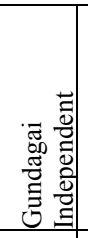 & 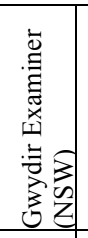 & 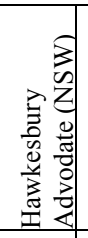 & 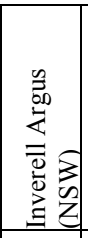 & 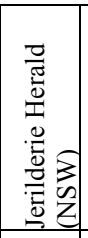 & 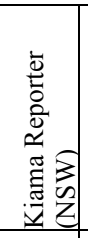 & 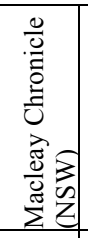 & 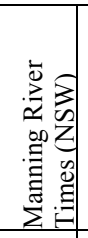 & 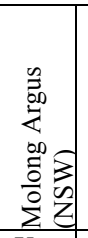 & 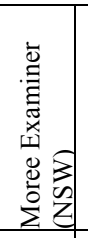 & 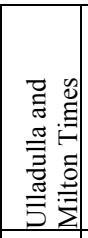 & 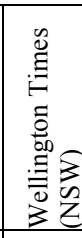 \\
\hline "Back to Life" (1897) & Unknown & Unk & $\mathrm{X}$ & $\mathrm{X}$ & & & & $\mathrm{X}$ & & & & & & & & & & & & \begin{tabular}{l|l}
$\mathrm{X}$ & $\mathrm{y}$ \\
\end{tabular} & & & \\
\hline "John Horton's Ward" (1897) & Grosvenor Bunster & Aust & & $\mathrm{X}$ & $\mathrm{X}$ & & & $\mathrm{X}$ & $\mathrm{X}$ & $\mathrm{X}$ & & & & & & & & & & $\mathrm{X}$ & & & \\
\hline "Right Wins" (1897) & Unknown & Unk & & $\mathrm{X}$ & & & & $\mathrm{X}$ & & & & & & & & & & & & $\mathrm{X}$ & & & \\
\hline "A Precious Villain" (1898) & Grosvenor Bunster & Aust & $\mathrm{X}$ & $\mathrm{X}$ & & $\mathrm{X}$ & & $\mathrm{X}$ & & & & & $\mathrm{X}$ & & & & & & $\mathrm{X}$ & $\mathrm{X}$ & & & \\
\hline "Fortune's Freaks" (1898) & \# Captain East Gray & Unk & $\mathrm{X}$ & $\mathrm{X}$ & & $\mathrm{X}$ & & $\mathrm{X}$ & & & & & $\mathrm{X}$ & & & & & & $\mathrm{X}$ & $\mathrm{X}$ & & & \\
\hline "Lost and Won" (1898/9) & Unknown & Unk & $\mathrm{X}$ & $\mathrm{X}$ & & & & $\mathrm{X}$ & & & & $\mathrm{X}$ & $\mathrm{X}$ & & & & & $\mathrm{X}$ & $\mathrm{X}$ & $\mathrm{X}$ & & & \\
\hline "Davies the Bushranger" (1899) & James J. Wright & Aust & $\mathrm{X}$ & $\mathrm{X}$ & & & & $\mathrm{X}$ & & & $\mathrm{X}$ & $\mathrm{X}$ & $\mathrm{X}$ & & $\mathrm{X}$ & $\mathrm{X}$ & $\mathrm{X}$ & $\mathrm{X}$ & $\mathrm{X}$ & $\mathrm{X}$ & & & \\
\hline "The Fatal Lilies" (1899) & Charlotte Brame & Brit & & $\mathrm{X}$ & & & $\mathrm{X}$ & $\mathrm{X}$ & & & $\mathrm{X}$ & $\mathrm{X}$ & $\mathrm{X}$ & & $\mathrm{X}$ & $\mathrm{X}$ & $\mathrm{X}$ & $\mathrm{X}$ & $\mathrm{X}$ & $\mathrm{X}$ & & $\mathrm{X}$ & \\
\hline $\begin{array}{l}\text { "The Last of the Pirates; Or, Doom Driven: A } \\
\text { Romance of the End of Ocean Outlawry" (1899) }\end{array}$ & Colonel Prentiss Ingraham & $\mathrm{Am}$ & & $\mathrm{X}$ & & & $\mathrm{X}$ & $\mathrm{X}$ & & & $\mathrm{X}$ & & $\mathrm{X}$ & $\mathrm{X}$ & $\mathrm{X}$ & $\mathrm{X}$ & $\mathrm{X}$ & $\mathrm{X}$ & $\mathrm{X}$ & $\mathrm{X}$ & $\mathrm{X}$ & & $\mathrm{X}$ \\
\hline
\end{tabular}

\section{Syndicate 11}

\begin{tabular}{|c|c|c|c|c|c|c|c|c|c|c|c|c|c|c|c|c|c|c|c|c|c|c|}
\hline Title & Author & Nat. & 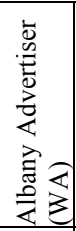 & 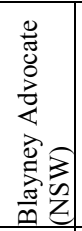 & 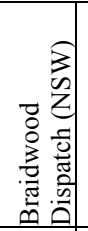 & 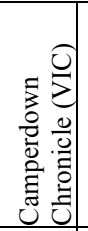 & 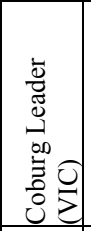 & 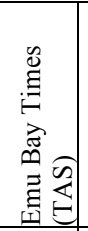 & 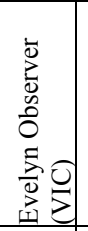 & 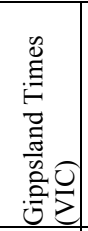 & 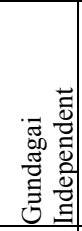 & 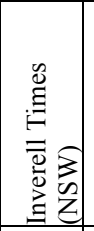 & 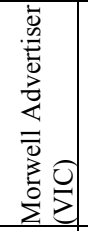 & 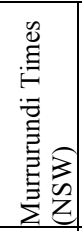 & 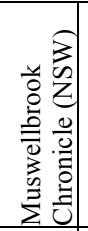 & 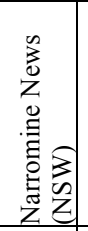 & 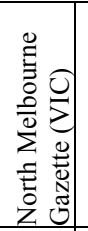 & 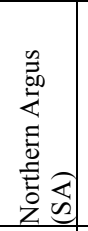 & 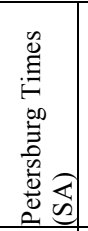 & 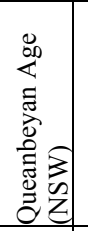 & 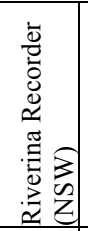 & 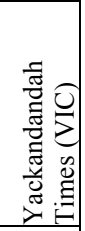 \\
\hline "The Chevalier de Maison Rouge" (1897/8) & Alexander Dumas & Fre & & & $\mathrm{X}$ & $\mathrm{X}$ & & & $\mathrm{X}$ & & & & $\mathrm{X}$ & $\mathrm{X}$ & $\mathrm{X}$ & & & & & & & $\mathrm{X}$ \\
\hline "More than a Friend" (1898) & Maggie Robertson & Brit & & & $\mathrm{X}$ & & & & & & $\mathrm{T}$ & & & & $\mathrm{X}$ & & & & & $\mathrm{X}$ & & \\
\hline "Parting of the Veil" (1898) & Everett W. Fish & $\mathrm{Am}$ & & & $\mathrm{X}$ & & & $\mathrm{X}$ & $\mathrm{X}$ & & & & $\mathrm{X}$ & $\mathrm{X}$ & $\mathrm{X}$ & & & $\mathrm{X}$ & $\mathrm{X}$ & $\mathrm{X}$ & & $\mathrm{X}$ \\
\hline "The Storm" (1898) & Armand Sylvestre & Fre & & & $\mathrm{X}$ & & & & & & & & & $\mathrm{X}$ & $\mathrm{X}$ & & & & & & & \\
\hline "The Testimony of Esther Sandys" (1898) & Roma White & Brit & & & $\mathrm{X}$ & & $\mathrm{X}$ & & & & $\mathrm{X}$ & $\mathrm{X}$ & & $\mathrm{X}$ & $\mathrm{X}$ & $\mathrm{X}$ & & & & & $\mathrm{X}$ & \\
\hline "Lost and Found" (1899) & Robert P. Whitworth & Aust & $\mathrm{X}$ & & & & & & $\mathrm{X}$ & & & & & & & & $\mathrm{X}$ & $\mathrm{X}$ & $\mathrm{X}$ & & $\mathrm{X}$ & \\
\hline "What Gold Cannot Buy" (1899) & Mrs Alexander & Brit & & & & & & & $\mathrm{T}$ & $\mathrm{X}$ & & & & & & & $\mathrm{X}$ & $\mathrm{X}$ & $\mathrm{X}$ & & & \\
\hline "In the Wake of Fortune" (1899) & James J. Wright & Aust & & & & $\mathrm{X}$ & & & $\mathrm{X}$ & & & & & & & & & $\mathrm{X}$ & $\mathrm{X}$ & & & \\
\hline "Polly the Tea-Room Girl" (1899) & David Pae & Brit & & $\mathrm{X}$ & $\mathrm{X}$ & & $\mathrm{X}$ & & & & $\mathrm{T}$ & $\mathrm{X}$ & & $\mathrm{X}$ & $\mathrm{X}$ & & & & & & $\mathrm{X}$ & \\
\hline
\end{tabular}

